\title{
Models for Mirror Symmetry Breaking via $\beta$-Sheet - Controlled Copolymerization: (i) Mass Balance and (ii) Probabilistic Treatment
}

\author{
Celia Blanco, ${ }^{1, *}$ and David Hochberg ${ }^{1,0}$ \\ ${ }^{1}$ Centro de Astrobiología (CSIC-INTA), Carretera Ajalvir Kilómetro 4, 28850 Torrejón de Ardoz, Madrid, Spain
}

\begin{abstract}
Experimental mechanisms that yield the growth of homochiral copolymers over their heterochiral counterparts have been advocated by Lahav and coworkers. These chiral amplification mechanisms proceed through racemic $\beta$-sheet controlled polymerization operative in both surface crystallites as well as in solution. We develop two complementary theoretical models for these template-induced desymmetrization processes leading to multi-component homochiral copolymers. First, assuming reversible $\beta$-sheet formation, the equilibrium between the free monomer pool and the polymer strand within the template is assumed. This yields coupled non-linear mass balance equations whose solutions are used to calculate enantiomeric excesses and average lengths of the homochiral chains formed. The second approach is a probabilistic treatment based on random polymerization. The occlusion probabilities depend on the polymerization activation energies for each monomer species and are proportional to the concentrations of the monomers in solution in the constant pool approximation. The monomer occlusion probabilities are represented geometrically in terms of unit simplexes from which conditions for maximizing or minimizing the likelyhood for mirror symmetry breaking can be determined.
\end{abstract}

\section{INTRODUCTION}

Mirror or chiral symmetry is broken in all known biological systems, where processes crucial for life such as replication, imply chiral supramolecular structures, sharing the same chiral sign (homochirality). These chiral structures are proteins, composed of aminoacids almost exclusively found as the left-handed enantiomers (S), also DNA, and RNA polymers and sugars with chiral building blocks composed by right-handed $(\mathrm{R})$ monocarbohydrates. The emergence of this biological homochirality in the chemical evolution from prebiotic to living systems is an enticing enigma in the origin of life and early evolution and is a compelling problem that foments scientific activity transcending the traditional boundaries of physics, chemistry and biology [1].

Biological homochirality of living systems involves large macromolecules, therefore a key issue is the relationship of the polymerization process with the emergence of chirality. This problem has generated activity in theoretical modeling aimed at understanding mirror symmetry breaking in chiral polymerization. Most of the models proposed [2, 4-10] can be understood as elaborated extensions and generalizations of Frank's original paradigmatic scheme [3]. An early work is that of Sandars [2], who introduced a detailed polymerization process plus the basic elements of enantiomeric cross inhibition as well as a chiral feedback mechanism in which only the largest polymers formed can enhance the production of the monomers from an achiral substrate. He provided basic numerical studies of symmetry breaking and bifurcation properties of this model for various values of the number of repeat units $N$. The subsequent models cited below are actually variations on Sandars' original theme. Thus, Brandenburg and coworkers [5] studied the stability and conservation properties of a modified Sandars' model and introduce a reduced $N=2$ version including the effects of chiral bias. They included spatial extent [4] in this model to study the spread and propagation of chiral domains as well as the influence of a backround turbulent advection velocity field. The model of Wattis and Coveney [6] differs from Sandars' in that they allow for polymers to grow to arbitrary lengths $N$ and the chiral polymers of all lengths, from the dimer and upwards, act catalytically in the breakdown of the achiral source into chiral monomers. An analytic linear stability analysis of both the racemic and chiral solutions is carried out for the model's large $N$ limit and various kinetic timescales are identified. The role of external white noise on Sandars-type polymerization networks including spatial extent has been explored by Gleiser and coworkers: the $N=2$ truncated model cited above [4] is subjected to external white noise [7], chiral bias is then considered [8], as well as the influence of high intensity and long duration noise 9 . Modified Sandars-type models with spatial extent and external noise [10] are considered, allowing for both finite and infinite $N$, with an emphasis paid to the dynamics of chiral symmetry breaking. On the experimental side, Luisi et al. [11, 12] reported the polymerization of racemic tryptophan, leucine, or isoleucine in buffered solutions which yielded libraries of short oligopeptides in the range of

\footnotetext{
*Electronic address: blancodtc@cab.inta-csic.es
}

$\dagger$ Electronic address: hochbergd@cab.inta-csic.es 
six to ten residues, where the isotactic peptides were formed as minor diastereoisomers, in amounts larger than those predicted by a purely random binomial distribution.

One scenario for the transition from prebiotic racemic chemistry to chiral biology suggests that homochiral peptides or amino acid chains must have appeared before the onset of the primeval enzymes [13 17]. However, except for a couple of known cases [11, 12], the polymerization of racemic mixtures (i.e., in 1:1 proportions) of monomers in ideal solutions typically yields chains composed of random sequences of both the left and right handed repeat units following a binomial distribution [1]. This statistical problem has been overcome recently by the experimental demonstration of the generation of amphiphilic peptides of homochiral sequence, that is, of a single chirality, from racemic compositions or racemates. This route consists of two steps: (1) the formation of racemic parallel or anti-parallel $\beta$-sheets either in aqueous solution or in 3-D crystals [19] during the polymerization of racemic hydrophobic $\alpha$-amino acids (Figure 10) followed by (2) an enantioselective controlled polymerization reaction [20 26]. This process leads to racemic or mirrorsymmetric mixtures of isotactic oligopeptides where the chains are composed from amino acid residues of a single handedness (see Figure 11). Furthermore, when racemic mixtures of different types of amino acids were polymerized, isotactic co-peptides of homochiral sequence were generated. The guest $(\mathrm{S})$ and $(\mathrm{R})$ molecules are enantioselectively incorporated into the chains of the $(\mathrm{S})$ and $(\mathrm{R})$ peptides, respectively, however the guest molecules are randomly distributed within the corresponding homochiral chains, see Figure 2 ,

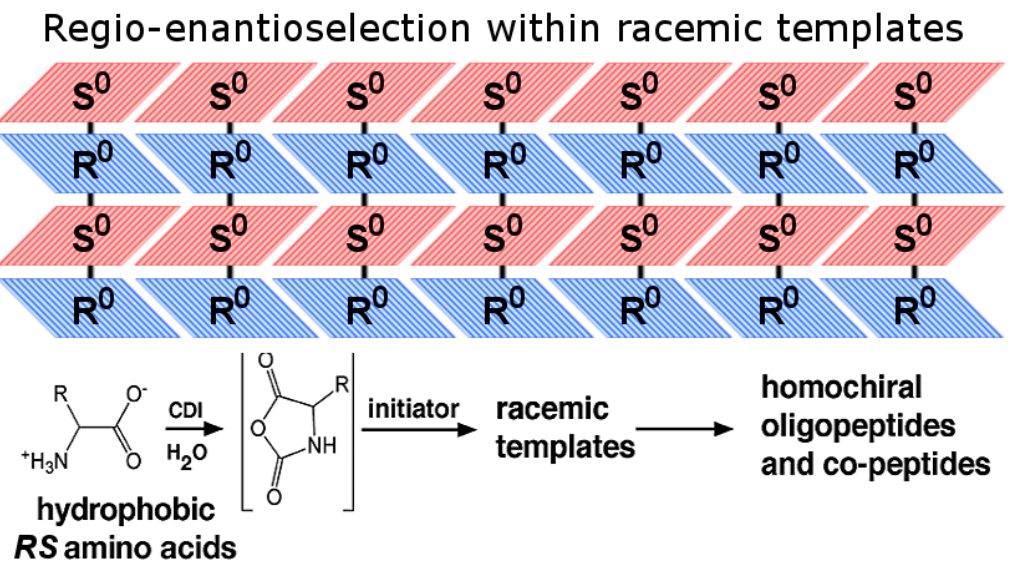

FIG. 1: Self assembly of oligopeptides into racemic $\beta$-sheets, for the case of a single species $\left(R_{0}, S_{0}\right)$ of amino acid supplied in ideally racemic proportions. For a full experimental account, see Weissbuch et al. 19].

As a combined result of these two steps, the sequence of pairs of co-peptide $\mathrm{S}$ and $\mathrm{R}$ chains within the growing template will differ from each other, see Figure 2. This results in non-racemic mixtures of co-peptide polymer chains of different sequences. Consequently, by considering the sequences of the peptide chains, a statistical departure from the racemic composition of the library of the peptide chains is created which varies with chain length and with the relative concentrations of the monomers used in the polymerization [21, 22]. This can be appreciated comparing Figure 1] and Figure 2 in the former the $\beta$-sheet is globally racemic (no guest amino acids) whereas the latter template is not by virtue of the randomness of the specific amino acid sequences within each homochiral strand, due to the presence of guest amino acids. It is precisely here, in the $\beta$ - sheet template, that mirror symmetry is stochastically broken. Non-enantiomeric pairs of homochiral chains are formed; this mechanism relies crucially on the presence of more than one type of amino acid. Note that this does not necessarily imply any net optical activity of solution containing the remaining free chiral monomers.

In this paper we report a theoretical investigation of multi-component copolymerization controlled by such templates. The models we introduce presuppose or take as given the prior formation of the initial templates or $\beta$-sheets and is concerned exclusively with the subsequent enantioselective polymerization reactions. Thus the nonlinear template control is implicit throughout our discussions. We consider two distinct model approaches to the problem. The first is based on detailed balance where the polymerization proceeds through stepwise isodesmic additions and dissociations of the chiral monomers (amino acids) to one end of the growing homochiral chain within the template. This can be treated knowing the compositions of the majority and minority monomers and their associated equilibrium constants, and we use chemical kinetics at equilibrium as a useful approximation to completely solve the problem. In thermodynamic equilibrium detailed balance allows us to derive coupled sets of nonlinear mass balance equations. Their solutions yield the equilibrium concentrations of all the monomers and chiral copolymers in terms of the equilibrium constants and the initial total monomer compositions. With these in hand, we can calculate the enantiomeric excesses of the homochiral chains and their average lengths. The degree of mirror symmetry breaking 
depends on the numbers of the monomer components and their relative concentrations [27]. A brief communication of preliminary results obtained from this equilibrium model were reported recently by the authors [27]. This paper extends and generalizes that previous work.

The second model approach is based on strictly probabilistic or statistical considerations and does not assume chemical equilibrium. We will consider the general case involving racemic mixtures of various species of enantiomers, that is, a variety of racemic guest molecules, which can occlude randomly into the chiral sites of the host racemic $\beta$-sheet or crystal site [21, 22, 25, 26]. Among the questions to be addressed: how many species $m$ of such guests are needed to break mirror symmetry? How many repeat units $N$ should the homochiral chains have? What are the ideal mole fractions of the monomers in solution for symmetry breaking? To answer these questions we first calculate the probability that a given homochiral sequence is formed from the majority and minority species. For random copolymerization, the attachment probability, or the probability of occlusion by the template/crystal, of an amino acid monomer to the growing chain is proportional to its concentration in solution, and we will invoke the constant pool approximation. This probability depends on the polymerization activation energy of the individual monomers (through the Arrhenius relation). The second part deals with combinatorics: counting the number of rearrangements of a given sequence, as all these independent sequences or "re-shufflings" will have the same probability to form. The information from both these parts permits us to calculate the joint probability of finding enantiomeric pairs and from this we deduce the net probability for finding non-enantiomeric pairs. The latter provides a statistical measure of the likelihood that mirror symmetry is broken as a function of chain length and the number and concentration of the minority species. We will then generalize these arguments to the case of many additives, and even allow nonracemic initial concentrations for all the amino acid species. Here again, the underlying kinetic template control is assumed implicitly.

Both approaches assume that the template-controlled polymerization obeys a first-order Markov process. Experiments carried out in solution [11, 12] appear to confirm this expectation: these results were subsequently rationalized by a mathematical model assuming a first-order Markov mechanism [28].

These two theoretical perspectives afford a complementary view of the induced mirror symmetry breaking scenario as originally proposed by Lahav and coworkers. The first scenario holds for closed systems in equilibrium where the monomers are depleted/replenished by the polymerization. We can nevertheless approximate irreversible polymerization as well as we please by simply choosing sufficiently large equilibrium constants. The numerical effects are negligible. The second approach is apt for open systems where the monomer pool is held constant and is free from the assumption of equilibrium.

\section{THEORETICAL METHODS I}

\section{A. Mass Balance}

To address the general setting for the generation of libraries of diastereoisomeric mixtures of peptides as originally proposed by Nery at al. 21], we need a suitable generalization of their scenario. To this end we consider the case where we have a majority amino acid species $\left(R_{0}, S_{0}\right)$ and a given number $m \geq 1$ of minority amino acid species $\left(R_{1}, S_{1}\right),\left(R_{2}, S_{2}\right), \ldots\left(R_{m}, S_{m}\right)$. Since the following calculations are based on chemical equilibrium and detailed balance, if all $(m+1)$ species are supplied in strictly 1:1 racemic proportions, we would justifiably expect a racemic outcome, that is, no mirror symmetry breaking. However, we can test the model's ability for chiral amplification by considering unequal initial proportions for the $m$ minority species in solution. That is, does the enantiomeric excess ee increase as a function of chain length, and is it greater than the initial ee of the monomers? The three monomer case originally treated[21] is a specific example of this for $m=1$ and with $R_{1}=0$, that is the system contains $R_{0}, S_{0}$ and only the enantiomer $S_{1}$ of the guest species. We assume as given the prior formation of the initial templates or $\beta$-sheets, and are concerned exclusively with the subsequent enantioselective random polymerization reactions (step (2)). The underlying nonlinear template control is implicit throughout the discussion. We consider stepwise additions and dissociations of single monomers from one end of the (co)polymer chain, considered as a strand within the $\beta$-sheet, see Figure 2, It is reasonable to regard the $\beta$-sheet in equilibrium with the free monomer pool[32] $*$.

From detailed balance, each individual monomer attachment or dissociation reaction is in equilibrium. This holds

[43] * Ref. [32] reports a stochastic simulation of two concurrent orthogonal processes: 1) an irreversible condensation of activated amino acids and 2) reversible formation of racemic $\beta$-sheets of alternating homochiral strands. The two steps taken together comprise a twodimensional formulation of the problem. These architectures lead to the formation of chiral peptides whose isotacticity increases with length. 
for closed equilibrium systems in which the free monomers are depleted/replenished by the templated polymerization. Then we can compute the equilibrium concentrations of all the (co)-polymers in terms of equilibrium constants $K_{i}$ for each individual amino acid and the free monomer concentrations. The equilibrium concentration of an $S$-type copolymer chain of length $n_{0}+n_{1}+n_{2}+\ldots+n_{m}=N$ made up of $n_{j}$ molecules type $S_{j}$ is given by $p_{n_{0}, n_{1}, \ldots, n_{m}}^{S}=\left(K_{0} s_{0}\right)^{n_{0}}\left(K_{1} s_{1}\right)^{n_{1}} \ldots\left(K_{m} s_{m}\right)^{n_{m}} / K_{0}$, where $s_{j}=\left[S_{j}\right]$ [29]. Similarly for the concentration of an $R$-type copolymer chain of length $n_{0}^{\prime}+n_{1}^{\prime}+n_{2}^{\prime}+\ldots+n_{m}^{\prime}=N$ made up of $n_{j}^{\prime}$ molecules of type $R_{j}$ : $p_{n_{0}^{\prime}, n_{1}^{\prime}, \ldots, n_{m}^{\prime}}^{R}=\left(K_{0} r_{0}\right)^{n_{0}^{\prime}}\left(K_{1} r_{1}\right)^{n_{1}^{\prime}} \ldots\left(K_{m} r_{m}\right)^{n_{m}^{\prime}} / K_{0}$, where $r_{j}=\left[R_{j}\right]$. Note that we are considering only copolymers with random sequences such as $R 0-R 0-R 1-R 0-R 0-R 2-R 0-\ldots$ and $S 0-S 0-S 1-S 1-S 0-S 2-S 0-\ldots$. , but not heterochiral polymers (that is, no sequences involving both the $\mathrm{S}$ and $\mathrm{R}$ type monomers.) The equilibrium concentration equations we write down $p_{n_{0}, n_{1}, \ldots, n_{m}}^{S}, p_{n_{0}^{\prime}, n_{1}^{\prime}, \ldots, n_{m}^{\prime}}^{R}$ implicitly assume the underlying template control.

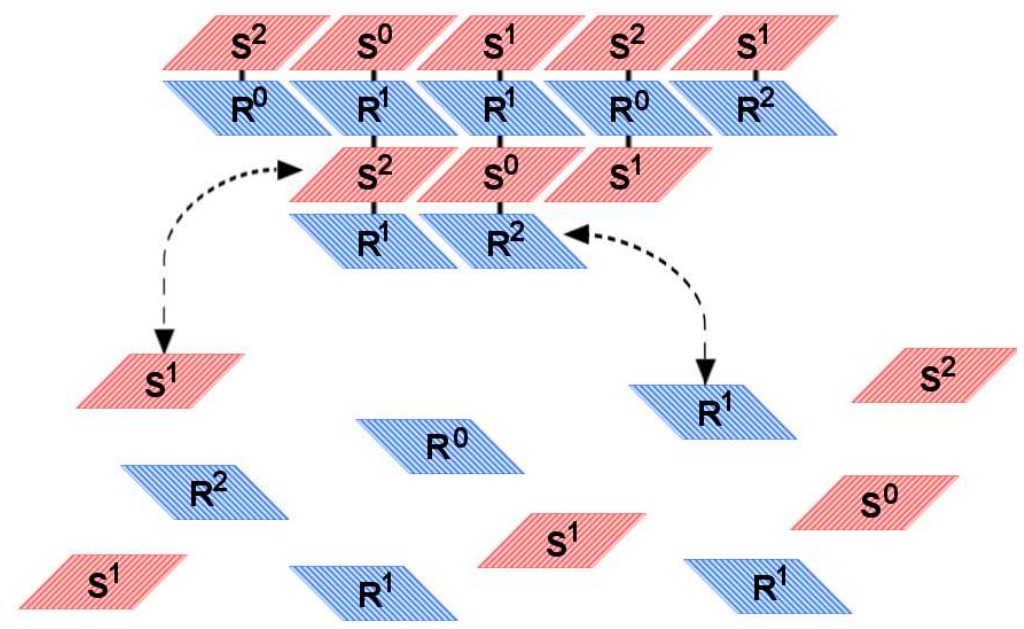

FIG. 2: The proposed scheme leading to enantioselective occlusion within racemic $\beta$-sheet templates. For the case illustrated, a majority host species $\left(R_{0}, S_{0}\right)$ and two minority guest species $\left(R_{1}, S_{1}\right)$ and $\left(R_{2}, S_{2}\right)$ of amino acids all of which are provided in ideally racemic proportions. The amino acids of a given chirality attach to sites of the same handedness within the growing $\beta$ sheet leading to the polymerization of oligomer strands of a uniform chirality, and in the alternating row $S-R-S-R-\ldots$ fashion as depicted. Since the polymerization in any given row is random and the guest monomers are typically less abundant than the host species, the former will occlude in a random way leading to independent uncorrelated random sequences in each chiral strand. The overall process yields non-enantiomeric pairs of homochiral copolymers, so that mirror symmetry is broken in a stochastic manner. The corresponding mass balance equations Eq. [5] are obtained assuming the monomer attachment/dissociation is in chemical equilibrium.

The number of different $S$-type copolymers of length $l$ with $n_{j}$ molecules of type $S_{j}$, for $0 \leq j \leq m$ species, is given by the multinomial coefficient:

$$
\left(\begin{array}{c}
l \\
n_{0}, n_{1}, \ldots, n_{m}
\end{array}\right)=\frac{l !}{n_{0} ! n_{1} ! \ldots n_{m} !} .
$$

Hence the total concentration of the $S$-type copolymers of length $l$ within the $\beta$-sheet is given by

$$
p_{l}^{S}=\sum_{n_{0}+n_{1}+\ldots+n_{m}=l}\left(\begin{array}{c}
l \\
n_{0}, n_{1}, \ldots, n_{m}
\end{array}\right) p_{n_{0}, n_{1}, \ldots, n_{m}}^{S}=\frac{1}{K_{0}}\left(K_{0} s_{0}+K_{1} s_{1}+\ldots+K_{m} s_{m}\right)^{l},
$$

which follows from the multinomial theorem [30]. From this we can calculate the number of each type $S_{j}$ of $S$-monomer present in the $S$-copolymer of length equal to $l$, for any $0 \leq j \leq m$ :

$$
\begin{aligned}
s_{j}\left(p_{l}^{S}\right) & =\sum_{n_{0}+n_{1}+\ldots+n_{m}=l}\left(\begin{array}{c}
l \\
n_{0}, n_{1}, \ldots, n_{m}
\end{array}\right) n_{j} p_{n_{0}, n_{1}, \ldots, n_{m}}^{S}=s_{j} \frac{\partial}{\partial s_{j}} p_{l}^{S} \\
& =\frac{K_{j}}{K_{0}} s_{j} l\left(K_{0} s_{0}+K_{1} s_{1}+\ldots+K_{m} s_{m}\right)^{l-1} .
\end{aligned}
$$


Then we need to know the total amount of the $S$-type monomers bound within the $S$-copolymers (in the $\beta$-sheet) from the dimer on up to a maximum chain length $N$. Using Eq. (3) for the $j$ th type of amino acid, this is given by

$$
s_{j}\left(p_{T o t}^{S}\right)=\sum_{l=2}^{N} s_{j}\left(p_{l}^{S}\right) \rightarrow \frac{K_{j}}{K_{0}} s_{j} \frac{a(2-a)}{(1-a)^{2}},
$$

the final expression holds in the limit $N \rightarrow \infty$ provided that $a=\left(K_{0} s_{0}+K_{1} s_{1}+\ldots+K_{m} s_{m}\right)<1$. This must be the case, otherwise the system would contain an infinite number of molecules [29]. Similar considerations hold for the $R$-sector, and the total amount of $R$ monomers inside $R$ copolymers for the $j t h$ amino acid, is given by $r_{j}\left(p_{T o t}^{R}\right)=\frac{K_{j}}{K_{0}} r_{j} \frac{b(2-b)}{(1-b)^{2}}$ where $b=\left(K_{0} r_{0}+K_{1} r_{1}+\ldots+K_{m} r_{m}\right)<1$. From this we obtain the mass balance equations which hold for both enantiomers $S, R$ of the host and guest amino acids, and is our key result [27]:

$$
s_{j}+\frac{K_{j}}{K_{0}} s_{j} \frac{a(2-a)}{(1-a)^{2}}=s_{j_{t o t}}, \quad r_{j}+\frac{K_{j}}{K_{0}} r_{j} \frac{b(2-b)}{(1-b)^{2}}=r_{j_{t o t}} .
$$

These equations express the fact that each type of enantiomer is either free in solution, or is else bound inside a (co)polymer strand within the template.

The problem then consists in the following: given the total concentrations of all the $m+1$ host plus guest enantiomers $\left\{s_{j_{\text {tot }}}, r_{j_{\text {tot }}}\right\}_{j=0}^{m}$, and the equilibrium constants $K_{i}$, we calculate the free monomer concentrations in solution $\left\{s_{j}, r_{j}\right\}_{j=0}^{m}$ from solving the nonlinear equations Eqs. (5). Denote by $s_{0_{t o t}}+\ldots+s_{m_{\text {tot }}}+r_{0_{\text {tot }}}+\ldots+r_{m_{\text {tot }}}=c_{\text {tot }}$ the total system concentration. From the solutions of Eq. (5) we can calculate e.g., the equilibrium concentrations of homochiral copolymers $p_{n_{0}, n_{1}, \ldots, n_{m}}^{S}$ and $p_{n_{0}^{\prime}, n_{1}^{\prime}, \ldots, n_{m}^{\prime}}^{R}$ of any specific sequence or composition as well as the resultant enantiomeric excess for homochiral chains of length $l$ composed of the host (majority) amino acid:

$$
e e_{l}=\frac{\left(r_{0}\right)^{l}-\left(s_{0}\right)^{l}}{\left(r_{0}\right)^{l}+\left(s_{0}\right)^{l}}
$$

At this juncture it is important to point out that our above approach assumes that the polymerization reactions are under thermodynamic control. If there are any kinetic effects, they will not be seen as they would contribute to the chain compositions at shorter (finite) time scales. Our aim here is to obtain the compositions at asymptotically long relaxation times, and we thus hypothesize that the dominant pathways are under thermodynamic control.

\section{B. Average chain lengths}

We can calculate the average copolymer chain lengths as functions of initial monomer compositions $s_{j t o t}, r_{j}$ tot and the equilibrium constants $K_{j}$, using the solutions of our mass balance equations Eq. (5).

The ensemble-averaged chain lengths afford an alternative measure of the degree of mirror symmetry breaking resulting from the desymmetrization process discussed in Nery el al.[21]. There are a number of relevant and interesting averages one can define and calculate. The average chain lengths, starting from the dimers, of the $S$-type copolymers, composed of random sequences of the $S_{j}$ type monomers, and that of the $R$-type copolymers composed of random sequences of the $R_{j}$ type monomers are given by:

$$
\begin{aligned}
& <l_{S}>=\frac{\sum_{l=2}^{N}\left(s_{0}\left(p_{l}^{S}\right)+s_{1}\left(p_{l}^{S}\right)+\ldots+s_{m}\left(p_{l}^{S}\right)\right)}{\sum_{l=2}^{N} p_{l}^{S}} \rightarrow \frac{\left(s_{0}+\frac{K_{1}}{K_{0}} s_{1}+\ldots+\frac{K_{m}}{K_{0}} s_{m}\right) \frac{a(2-a)}{(1-a)^{2}}}{\frac{a^{2}}{(1-a) K_{0}}}=\left(\frac{2-a}{1-a}\right) \\
& <l_{R}>=\frac{\sum_{l=2}^{N}\left(r_{0}\left(p_{l}^{R}\right)+r_{1}\left(p_{l}^{R}\right)+\ldots+r_{m}\left(p_{l}^{R}\right)\right)}{\sum_{l=2}^{N} p_{l}^{R}} \rightarrow \frac{\left(r_{0}+\frac{K_{1}}{K_{0}} r_{1}+\ldots+\frac{K_{m}}{K_{0}} r_{m}\right) \frac{b(2-b)}{(1-b)^{2}}}{\frac{b^{2}}{(1-b) K_{0}}}=\left(\frac{2-b}{1-b}\right)
\end{aligned}
$$

respectively. We also obtain an expression for the average length of the polymer chains composed exclusively from sequences of the $S_{j}$ or $R_{j}$ enantiomers of a given specific amino acid of type $j$ :

$$
<l_{S}^{s_{j}}>=\frac{\sum_{l=2}^{N} s_{j}\left(p_{l}^{S\left(s_{j}\right)}\right)}{\sum_{l=2}^{N} p_{l}^{S\left(s_{j}\right)}}=\frac{\sum_{l=2}^{N} \frac{K_{j}}{K_{0}} s_{j} l\left(K_{j} s_{j}\right)^{l-1}}{\sum_{l=2}^{N} \frac{\left(K_{j} s_{j}\right)^{l}}{K_{0}}} \rightarrow \frac{\frac{\left(s_{j} K_{j}\right)^{2}\left(2-K_{j} s_{j}\right)}{\left(1-K_{j} s_{j}\right)^{2}}}{\frac{\left(K_{j} s_{j}\right)^{2}}{\left(1-K_{j} s_{j}\right)}}=\left(\frac{2-K_{j} s_{j}}{1-K_{j} s_{j}}\right),
$$


TABLE I: The definitions of the various average chain lengths $<l>,\left\langle l_{S}\right\rangle,\left\langle l_{R}\right\rangle,<l_{S}^{s_{j}}>$ and $<l_{R}^{r_{j}}>$ employed.

$<l>$ Average length of all the copolymers in the system

$\left\langle l_{S}>\right.$ Average length of all the $S$-type copolymers, composed of random sequences of the $S_{j}$ type monomers

$<l_{R}>$ Average length of all the $R$-type copolymers, composed of random sequences of the $R_{j}$ type monomers

$<l_{S}^{s_{j}}>$ Average length of the polymer exclusively composed from sequences of the $S_{j}$ enantiomers of a given amino acid type $j$

$<l_{R}^{r_{j}}>$ Average length of the polymer exclusively composed from sequences of the $R_{j}$ enantiomers of a given amino acid type $j$

$$
<l_{R}^{r_{j}}>=\frac{\sum_{l=2}^{N} r_{j}\left(p_{l}^{R\left(r_{j}\right)}\right)}{\sum_{l=2}^{N} p_{l}^{R\left(r_{j}\right)}}=\frac{\sum_{l=2}^{N} \frac{K_{j}}{K_{0}} r_{j} l\left(K_{j} r_{j}\right)^{l-1}}{\sum_{l=2}^{N} \frac{\left(K_{j} r_{j}\right)^{l}}{K_{0}}} \rightarrow \frac{\frac{\left(r_{j} K_{j}\right)^{2}\left(2-K_{j} r_{j}\right)}{\left(1-K_{j} r_{j}\right)^{2}}}{\frac{\left(K_{j} r_{j}\right)^{2}}{\left(1-K_{j} r_{j}\right)}}=\left(\frac{2-K_{j} r_{j}}{1-K_{j} r_{j}}\right) .
$$

To complete the list, we can calculate the chain length averaged over all the copolymers in the system:

$$
\begin{aligned}
<l> & =\frac{\sum_{l=2}^{N}\left(s_{0}\left(p_{l}^{S}\right)+s_{1}\left(p_{l}^{S}\right)+\ldots+s_{m}\left(p_{l}^{S}\right)+r_{0}\left(p_{l}^{R}\right)+r_{1}\left(p_{l}^{R}\right)+\ldots+r_{m}\left(p_{l}^{R}\right)\right)}{\sum_{l=2}^{N}\left(p_{l}^{S}+p_{l}^{R}\right)} \\
& \rightarrow \frac{\left(s_{0}+\frac{K_{1}}{K_{0}} s_{1}+\ldots+\frac{K_{m}}{K_{0}} s_{m}\right) \frac{a(2-a)}{(1-a)^{2}}+\left(r_{0}+\frac{K_{1}}{K_{0}} r_{1}+\ldots+\frac{K_{m}}{K_{0}} r_{m}\right) \frac{b(2-b)}{(1-b)^{2}}}{\frac{a^{2}}{(1-a) K_{0}}+\frac{b^{2}}{(1-b) K_{0}}} \\
& =\frac{a^{2}(2-a)(1-b)^{2}+b^{2}(2-b)(1-a)^{2}}{a^{2}(1-b)^{2}(1-a)+b^{2}(1-b)(1-a)^{2}} .
\end{aligned}
$$

The right-hand most expressions $(\rightarrow)$ in each case hold in the limit of $N \rightarrow \infty$ and for $a<1$ and $b<1$. See Table 【 for definitions of all these quantities.

\section{RESULTS}

\section{A. Induced desymmetrization}

We turn to the scenario discussed in Nery et al. 21] and consider the influence of a single guest species, so $m=1$ will be sufficient for our purposes. For a single guest, we drop numbered indices and denote the majority host species and concentrations by $r=[R], s=[S]$ and the minority guest with a prime: $s^{\prime}=\left[S^{\prime}\right]$.

We use the above framework to calculate the enantiomeric excess ee as a function of chain length $l$ for the three starting compositions of the monomer crystals as reported[21]. In Fig. 3 we plot the numerical results obtained from calculating Eq. 6, the only quantities required for this are the solutions of $r$ and $s$ obtained from solving the set of equations Eq.(5). For strictly illustrative purposes only, we set the equilibrium constants to be the same for both host and guest monomers $K_{1}=K_{0} \equiv K=1000 M^{-1}$, the total initial concentration, $c_{t o t}=10^{-3} M$; the initial fractions of each component are denoted by $f=\left\{f_{r}, f_{s}, f_{s^{\prime}}\right\}$ and obey $f_{r}+f_{s}+f_{s^{\prime}}=1$. The starting composition of the mixture is $c_{t o t}=r_{t o t}+s_{t o t}+s_{t o t}^{\prime}$, and the total amount of each component is: $r_{t o t}=c_{t o t} * f_{r}, s_{t o t}=c_{t o t} * f_{s}$, and $s_{\text {tot }}^{\prime}=c_{t o t} * f_{s^{\prime}}$. We can appreciate the induced symmetry breaking mechanism[21] from the behavior of $e e_{l}$. For the first case $f_{r}: f_{s}: f_{s^{\prime}}=0.5: 0.25: 0.25$, mirror symmetry is broken for almost all the chain lengths, even for small values of $l$ : for $l=3$ the $e e$ reaches $60 \%$ and for $l=5$ the $e e$ is found to be greater than $80 \%$, this is due to the equal starting fractions of the majority $s_{t o t}$ and the guest $s_{t o t}^{\prime}$ monomer species of the same chirality, the large amount of guest is the reason for these large values of ee. For the second case $f_{r}: f_{s}: f_{s^{\prime}}=0.5: 0.45: 0.05$, the starting fraction of the majority species, $s_{t o t}^{\prime}$, is almost 10 times $(0.45 / 0.05=9)$ greater than that of the guest, $s^{\prime}$, so for the enantiomeric excess to be greater than $60 \%$ the chain length must be at least $l=13$, and for obtaining an $e e$ of $80 \%$, the chain length must be at least $l=20$. Finally, for the third case, $f_{r}: f_{s}: f_{s^{\prime}}=0.5: 0.475: 0.025$, the starting fraction of the majority species, $s_{t o t}$, is almost 20 times $(0.475 / 0.025=19)$ greater than that of the guest, $s_{t o t}^{\prime}$, thus the enantiomeric excess for each chain length is expected to be much less than for the two previous cases, an $e e$ greater than $60 \%$ is found for the chain length $l=27$ and for reaching greater than $80 \%$, the chain length must be 
at least $l=42$. For the three cases, an increase of the $e e_{l}$ is observed (for all $l$ ) when increasing the starting fraction of the guest species, $s_{\text {tot }}^{\prime}$. When $s_{\text {tot }}^{\prime}$ is comparable to $s_{\text {tot }}$, while maintaining the proportion $R$-type:S-type=1:1, then symmetry breaking is ensured to be $>40 \%$ for all $l>5$.

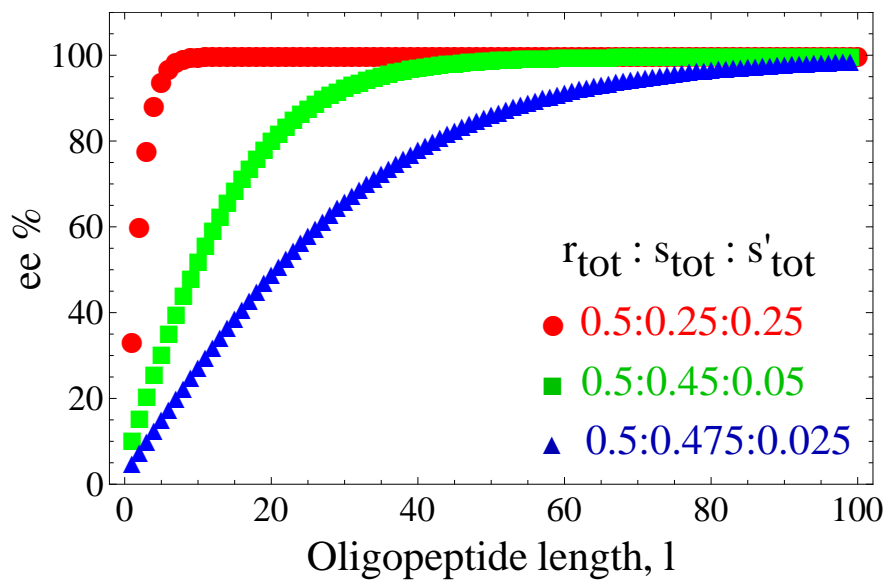

FIG. 3: Calculated ee values from solving Eqs. (5) for $m=1$ guest monomer and three different starting monomer compositions (in relative proportions) $r_{t o t}: s_{t o t}: s_{\text {tot }}^{\prime}=0.5: 0.25: 0.25$ (filled circles), $0.5: 0.45: 0.05$ (squares) and $0.5: 0.475: 0.025$ (triangles) for the equilibrium constant $K_{0}=K_{1}=1000 M^{-1}$ and the total monomer concentration $c_{t o t}=10^{-3} M$. Compare to Fig. 13 of Nery et al. [21].

The mass balance equations can be used for calculating the amount of free monomers in solution as well as the amounts of the monomers bound inside the polymers as functions of the starting compositions and $K$. Solving Eq.(5) yields the amounts of the free monomers, given by $\left(r, s, s^{\prime}\right)$, while the amounts of the $R, S$ and $S^{\prime}$ monomers inside the copolymers are given by the expressions $r_{\text {poly }}=r b(2-b) /(1-b)^{2}, s_{p o l y}=s a(2-a) /(1-a)^{2}$ and $s_{\text {poly }}^{\prime}=s^{\prime} a(2-a) /(1-a)^{2}$, respectively. Then the total amount of all the monomers in polymers is given by $c_{\text {poly }}=r_{\text {poly }}+s_{\text {poly }}+s_{\text {poly }}^{\prime}$, and the total amount of free monomers in solution is $c_{f r e e}=r+s+s^{\prime}$. In Fig. 4 we display the values of these quantities for the same three starting compositions considered above as a function of $c_{t o t}$ and for $K_{0}=K_{1}=1000 M^{-1}$. The first row of Fig. 4 indicates how the amount of free monomers in solution $c_{f r e e}$, is greater than the amount of those in polymers, $c_{\text {poly }}$, for values of $c_{t o t}$ below a critical value. Above this value, then $c_{\text {poly }}>c_{\text {free }}$ : that is, the majority of the monomers are to found in the polymers, not in solution. In the second row, the different contributions to $c_{\text {poly }}$ are plotted for each type of monomer. In the first case $s_{\text {tot }}: s_{\text {tot }}^{\prime}=1: 1$ and leads to $s_{\text {poly }}: s_{\text {poly }}^{\prime}=1: 1$ which is the most favorable case for mirror symmetry breaking. Increasing the starting ratio between $s_{\text {tot }}$ and $s_{\text {tot }}^{\prime}$, increases the difference between $s_{\text {poly }}$ and $s_{\text {poly }}^{\prime}$, and diminishes the degree of symmetry breaking. The curves for $s_{\text {poly }}$ approach that of $r_{\text {poly }}$ as $s_{\text {tot }}^{\prime}$ is diminished (from left to right in Fig. 4). Hence in the third case, where $s_{\text {tot }}: s_{\text {tot }}^{\prime}=0.475: 0.025$, almost all the monomers present in copolymers are the $S$ monomers. The same applies for the third row, where the different contributions to $c_{\text {free }}$ are plotted. Both the amounts of free monomers and those forming polymers increase when increasing $c_{t o t}$. The degree of mirror symmetry breaking can be visualized by the gap or vertical distance between the curves for $r_{\text {free }}$ and $s_{\text {free }}$ versus $c_{t o t}$ and as the amount of $s_{\text {free }}^{\prime}$ is varied. In a similar way Fig. 5] displays the same quantities for fixed $c_{t o t}=10^{-3} M$ and as functions of the equilibrium constant $K$. As before, $c_{p o l y}$ and its individual contributions all increase with increasing $K$, whereas $c_{f r e e}$ (and its individual contributions) all decrease. Clearly, increasing $K$ favors the formation of the polymers over their dissociation into free monomers, and we can approximate irreversible polymerization as close as we please by taking sufficiently large values of $K$.

The equilibrium concentration of the S-type copolymer chain of length $m+n=N$ made up of $m$ molecules $S$ and of $n$ molecules $S^{\prime}$ is given by $p_{m, n}^{S}=(K s)^{m}\left(K s^{\prime}\right)^{n} / K$. Using the solutions from Eqs. (5) we compute the mole fractions of each S-copolymer, normalized to its own subfamily as $\frac{p_{m, n}^{S}}{\sum_{m+n=N} p_{m, n}^{S}}$, this is displayed in Fig. 6 for $2 \leq N \leq 7$.

By way of one further example, we carry out a similar analysis for the case of four monomers, this time for two majority $R, S$ and two minority amino acids: $R^{\prime}, S^{\prime}$. From Eq. (6) we calculate the $e e_{l}$ for the different chain lengths $l$ for three different starting compositions. In Fig. [7] we show the numerical results obtained from the solutions of the set of equations Eq.(5) and Eq.([6), for $K_{0}=K_{1}=1000 M^{-1}$ and $s_{\text {tot }}+s_{\text {tot }}^{\prime}+r_{\text {tot }}+r_{\text {tot }}^{\prime}=10^{-3} M$.

As before, we can evaluate the mole fractions of both the $\mathrm{S}$ and R-type copolymers that are in equilibrium with the free monomer pool: namely $\frac{p_{m, n}^{S}}{\sum_{m+n=N} p_{m, n}^{S}}$ and $\frac{p_{m, n}^{R}}{\sum_{m+n=N} p_{m, n}^{R}}$, respectively. These are displayed in Fig. 8 for the 

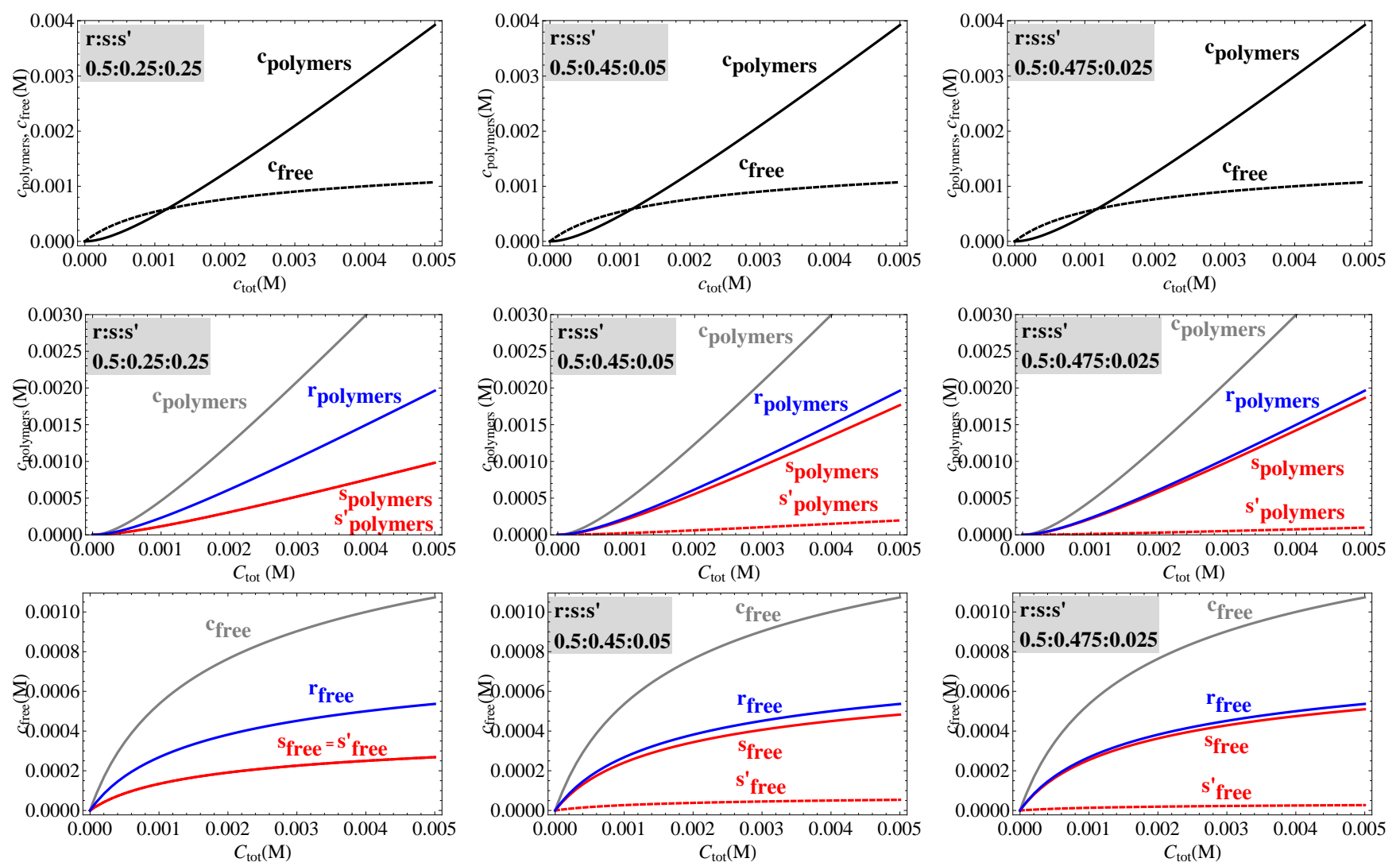

FIG. 4: $K_{0}=K_{1}=1000$. The amounts of free monomers and those bound in polymers as function of total monomer concentration $c_{\text {tot }}$ versus three different initial relative proportions: $r_{\text {tot }}: s_{\text {tot }}: s_{\text {tot }}^{\prime}=0.5: 0.25: 0.25, r_{\text {tot }}: s_{\text {tot }}: s_{\text {tot }}^{\prime}=0.5$ : $0.45: 0.05$ and $r_{t o t}: s_{t o t}: s^{\prime}{ }_{t o t}=0.5: 0.475: 0.025$. The first row: the total amount of free monomers and those forming polymers. Second row: the total and individual amounts of monomers forming polymers. Third row: the total and individual amounts of free monomers. See text for discussion.

initial total compositions indicated there.

Figures 3 and 7 clearly demonstrate that the higher (lower) is the initial degree of chiral asymmetry, characterized by $r_{t o t} / s_{t o t}$ in the former and $r_{t o t}^{\prime} / s_{t o t}^{\prime}$ in the latter, the higher (lower) is the final asymmetry. Thus, rather than symmetry breaking per se, we are observing the model's capacity for asymmetric amplification, as stated at the beginning of Section IIIA Nevertheless, effects closer to a symmetry breaking effect can be appreciated by looking at the average chain lengths for unequal equilibrium constants in Sec ШIB

\section{B. Average lengths of copolymer chains}

As an application of the mean chain length formulas derived in Eqs. (7611) in the following we focus on the simplest case of $m=1$ guest. We consider the effect of different equilibrium constants $K_{0} \neq K_{1}$ and a small total system concentration $c_{t o t}=10^{-3} \mathrm{M}$ in Table \. The dependence on varying $c_{t o t}$ for fixed but distinct equilibrium constants $K_{0} \neq K_{1}$ is displayed in Table $\amalg$.II.

Most interestingly, in Table 2 and 3, one can see the evolution of the global $r /\left(s+s^{\prime}\right)$ asymmetry, by looking at the $\left\langle l_{S}\right\rangle /\left\langle l_{R}\right\rangle$ difference. Especially from the results for the $0.5: 0.25: 0.25$ case, i.e. starting from a symmetric $r /\left(s+s^{\prime}\right)$ state, some chiral asymmetry, albeit small, is obtained between the length of the all-R and all-S copolymers. The source of this asymmetry is the ratio $K_{0} / K_{1}$ of the equilibrium constants, which we set to 2 in these examples. By contrast, when $K_{0}=K_{1}$ there is then no difference between $\left\langle l_{R}\right\rangle$ and $\left\langle l_{S}\right\rangle$. Conversely, greater ratios of $K_{0} / K_{1}$ lead to greater differences in $\left\langle l_{R}\right\rangle$ and $\left\langle l_{S}\right\rangle$ (data not shown).

Finally Tables [V] and $\mathrm{V}$ have been calculated for the same starting compositions as Figure 7 and can be compared with the latter. 

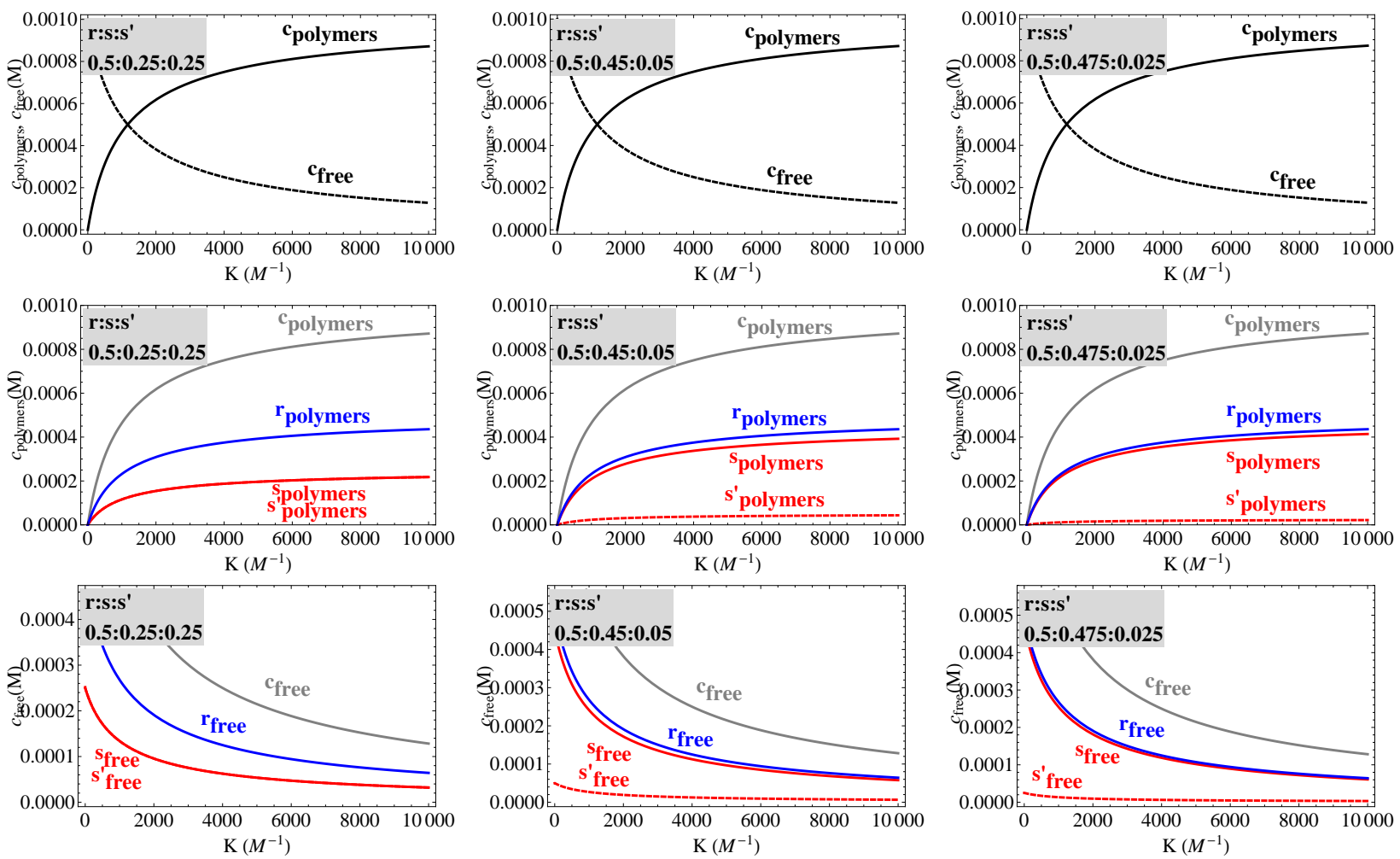

FIG. 5: $c_{t o t}=10^{-3}$. The amounts of free monomers and those in polymers as function of the equilibrium constant $K$ versus three different initial relative proportions: $r_{t o t}: s_{t o t}: s^{\prime}{ }_{t o t}=0.5: 0.25: 0.25, r_{t o t}: s_{t o t}: s^{\prime}{ }_{t o t}=0.5: 0.45: 0.05$ and $r_{\text {tot }}: s_{\text {tot }}: s^{\prime}{ }_{\text {tot }}=0.5: 0.475: 0.025$. The first row: the total amount of free monomers and those forming polymers. Second row: the total and individual amounts of monomers forming polymers. Third row: the total and individual amounts of free monomers. See text for discussion.

TABLE II: Average chain lengths for the three different starting compositions as a function of $K_{0}$ for $K_{1}=K_{0} / 2$ and $c_{\text {tot }}=10^{-3} \mathrm{M}$

\begin{tabular}{|c|c|c|c|c|c|c|c|c|c|c|c|c|c|c|c|}
\hline \multirow[b]{2}{*}{$K_{0}\left(M^{-1}\right)$} & \multicolumn{5}{|c|}{$r_{t o t}: s_{t o t}: s_{t o t}^{\prime}=0.5: 0.25: 0.25$} & \multicolumn{5}{|c|}{$r_{t o t}: s_{t o t}: s_{t o t}^{\prime}=0.5: 0.45: 0.05$} & \multicolumn{5}{|c|}{$r_{t o t}: s_{t o t}: s_{t o t}^{\prime}=0.5: 0.475: 0.025$} \\
\hline & $<l>$ & $<l_{S}>$ & $<l_{R}>$ & $<l_{S}^{s}>$ & $<l_{S}^{s^{\prime}}$ & $><l>$ & $<l_{S}>$ & $<l_{R}>$ & $\left.<l_{S}^{s}\right\rangle$ & $\left.<l_{S}^{s^{\prime}}\right\rangle$ & \rangle$\langle l\rangle$ & $<l_{S}>$ & $<l_{R}>$ & $\left.<l_{S}^{s}\right\rangle$ & $\left\langle l_{S}^{s^{\prime}}\right\rangle$ \\
\hline 1 & 2.00 & 2.00 & 2.00 & 2.00 & 2.00 & 2.00 & 2.00 & 2.00 & 2.00 & 2.00 & 2.00 & 2.00 & 2.00 & 2.00 & 2.00 \\
\hline 10 & 2.00 & 2.00 & 2.00 & 2.00 & 2.00 & 2.00 & 2.00 & 2.00 & 2.00 & 2.00 & 2.00 & 2.00 & 2.00 & 2.00 & 2.00 \\
\hline 00 & 2.04 & 2.04 & 2.05 & 2.02 & 2.01 & 2.05 & 2.05 & 2.05 & 2.04 & 2.00 & 2.05 & 2.05 & 2.05 & 2.04 & 2.00 \\
\hline 000 & 2.34 & 2.31 & 2.37 & 2.17 & 2.10 & 2.36 & 2.36 & 2.37 & 2.32 & 2.02 & 2.36 & 2.36 & 2.37 & 2.34 & 2.00 \\
\hline 10000 & 3.76 & 3.73 & 3.79 & 2.51 & 2.42 & 3.79 & 3.78 & 3.80 & 3.40 & 2.06 & 3.79 & 3.79 & 3.79 & 3.58 & 2.03 \\
\hline 100000 & 8.57 & 8.56 & 8.59 & 2.78 & 2.75 & 8.59 & 8.58 & 8.59 & 5.60 & 2.10 & 8.59 & 8.59 & 8.59 & 6.73 & 2.04 \\
\hline
\end{tabular}

TABLE III: Average chain lengths for the three different starting compositions as a function of $c_{t o t}$ for $K_{0}=100000$ and $K_{1}=K_{0} / 2$

\begin{tabular}{|c|c|c|c|c|c|c|c|c|c|c|c|c|c|c|c|}
\hline \multirow[b]{2}{*}{$\underline{c_{t o t}(M)}$} & \multicolumn{5}{|c|}{$r_{\text {tot }}: s_{\text {tot }}: s_{\text {tot }}^{\prime}=0.5: 0.25: 0.25$} & \multicolumn{5}{|c|}{$r_{\text {tot }}: s_{\text {tot }}: s_{\text {tot }}^{\prime}=0.5: 0.45: 0.05$} & \multicolumn{5}{|c|}{$r_{\text {tot }}: s_{\text {tot }}: s_{\text {tot }}^{\prime}=0.5: 0.475: 0.025$} \\
\hline & $<l>$ & $<l_{S}>$ & $<l_{R}>$ & $<l_{S}^{s}>$ & $\left.<l_{S}^{s^{\prime}}\right\rangle$ & $<l>$ & $\left.<l_{S}\right\rangle$ & $<l_{R}>$ & $<l_{S}^{s}>$ & $<l_{S}^{s^{\prime}}$ & $><l>$ & $\left.<l_{S}\right\rangle$ & $\left.<l_{R}\right\rangle$ & $<l_{S}^{s}>$ & $<l_{S}^{s^{\prime}}>$ \\
\hline $10^{-5}$ & 2.34 & 2.31 & 2.37 & 2.17 & 2.10 & 2.36 & 2.36 & 2.36 & 2.32 & 2.02 & 2.36 & 2.36 & 2.37 & 2.34 & 2.01 \\
\hline $10^{-4}$ & 3.76 & 3.73 & 3.79 & 2.51 & 2.42 & 3.79 & 3.78 & 3.79 & 3.40 & 2.06 & 3.79 & 3.79 & 3.79 & 3.58 & 2.03 \\
\hline $10^{-3}$ & 8.57 & 8.56 & 8.59 & 2.78 & 2.75 & 8.59 & 8.58 & 8.59 & 5.60 & 2.09 & 8.59 & 8.59 & 8.59 & 6.73 & 2.04 \\
\hline $10^{-2}$ & 23.86 & 23.86 & 23.87 & 2.92 & 2.91 & 23.87 & 23.86 & 23.87 & 8.18 & 2.11 & 23.87 & 23.87 & 23.87 & 11.93 & 2.05 \\
\hline $10^{-1}$ & 72.21 & 72.21 & 72.21 & 2.97 & 2.97 & 72.22 & 72.22 & 72.21 & 9.88 & 2.11 & 72.24 & 72.27 & 72.21 & 16.79 & 2.05 \\
\hline
\end{tabular}




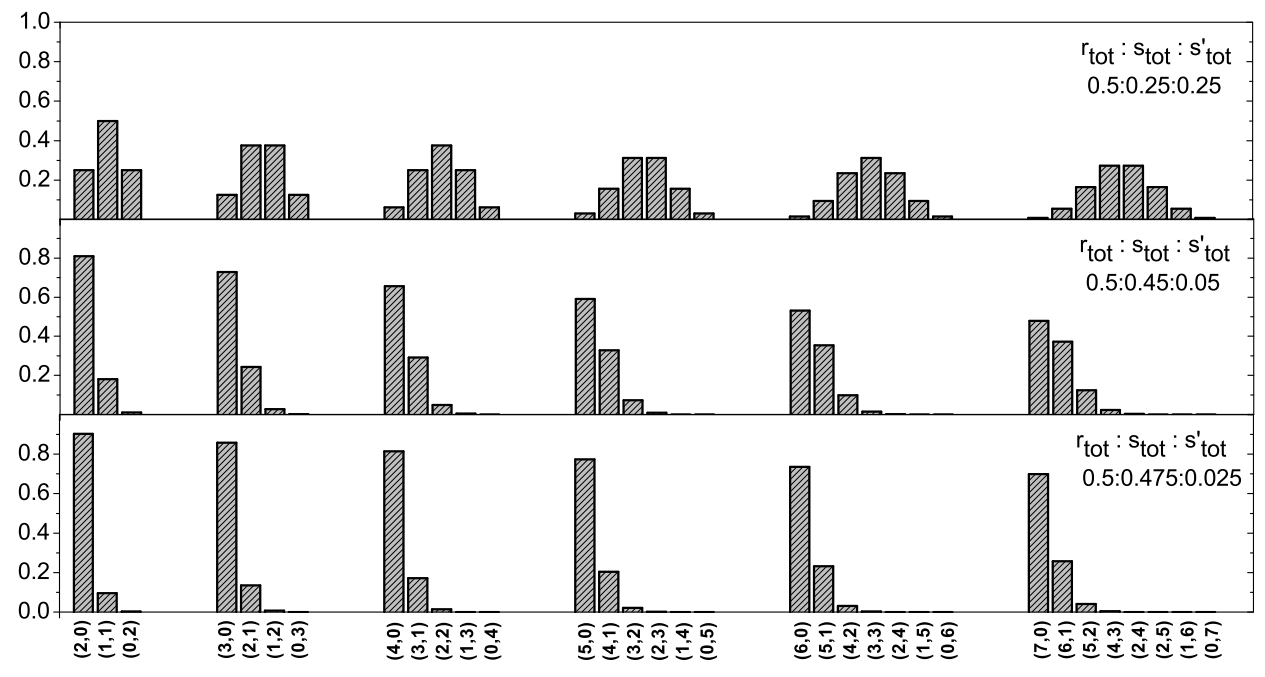

FIG. 6: Mole fractions of the S-type copolymers composed of $n S$ and $m S^{\prime}$ monomers for $m+n=N$ with $2 \leq N \leq 7$, as functions of the total initial fractions $r_{t o t}: s_{t o t}: s_{t o t}^{\prime}$. For $s_{t o t}: s_{\text {tot }}^{\prime}=1: 1$, the distributions are binomial (top), but when $s_{\text {tot }}: s_{\text {tot }}^{\prime}=9: 1$ (center) or when $s_{t o t}: s_{\text {tot }}^{\prime}=19: 1$ (bottom), then the distributions are skewed.

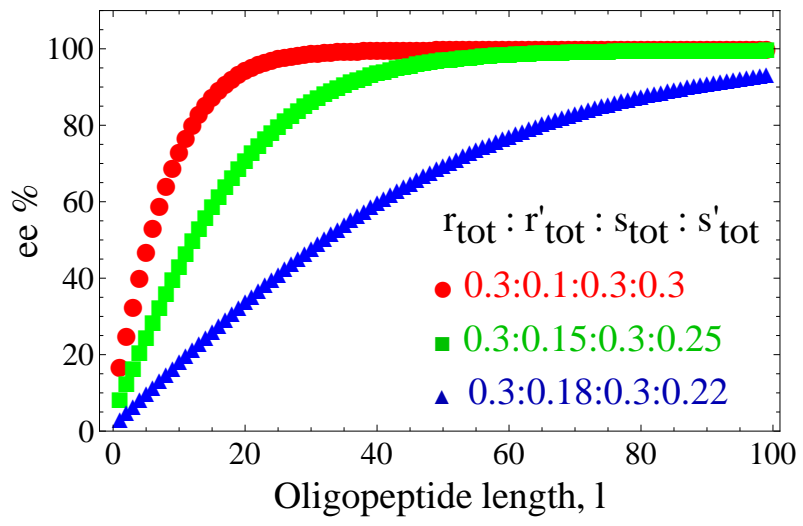

FIG. 7: Calculated ee values from solving Eqs. (5) for three different starting monomer compositions (in relative proportions) $r_{\text {tot }}: r_{\text {tot }}^{\prime}: s_{\text {tot }}: s_{\text {tot }}^{\prime}=0.3: 0.1: 0.3: 0.3$ (filled circles), $0.3: 0.15: 0.3: 0.25$ (squares) and $0.3: 0.18: 0.3: 0.22$ (triangles) for the equilibrium constant $K_{0}=K_{1}=1000 M^{-1}$ and the total monomer concentration $c_{\text {tot }}=10^{-3} \mathrm{M}$.

\section{THEORETICAL METHODS II}

\section{A. Probabilistic approach}

In the following sections, we adopt a statistical approach for calculating the likelihood for finding non-enantiomeric pairs of copolymers formed by the proposed template mechanism. This approach does not require chemical equilibrium. How many species $m$ of the chiral guest monomers are needed to break mirror symmetry? How many repeat units $N$ should the chains have? Are there conditions on the polymerization activation energies and mole fractions of the monomers in solution for maximizing the mirror symmetry breaking? We provide answers to these questions based on statistics, and this means being able to count polymer configurations, distinguishing sequences from compositions, and applying some basic combinatorial analysis. Indeed, we may regard the specific homochiral copolymerization sequences formed within the template mechanism as outcomes or "tosses" of generalized multifaceted "dies" (e.g., see Figure 22). However, these dies are loaded, in the sense that not all faces of the generalized die have an equal probability of turning up in any given throw. This is because different amino acids have different polymerization 

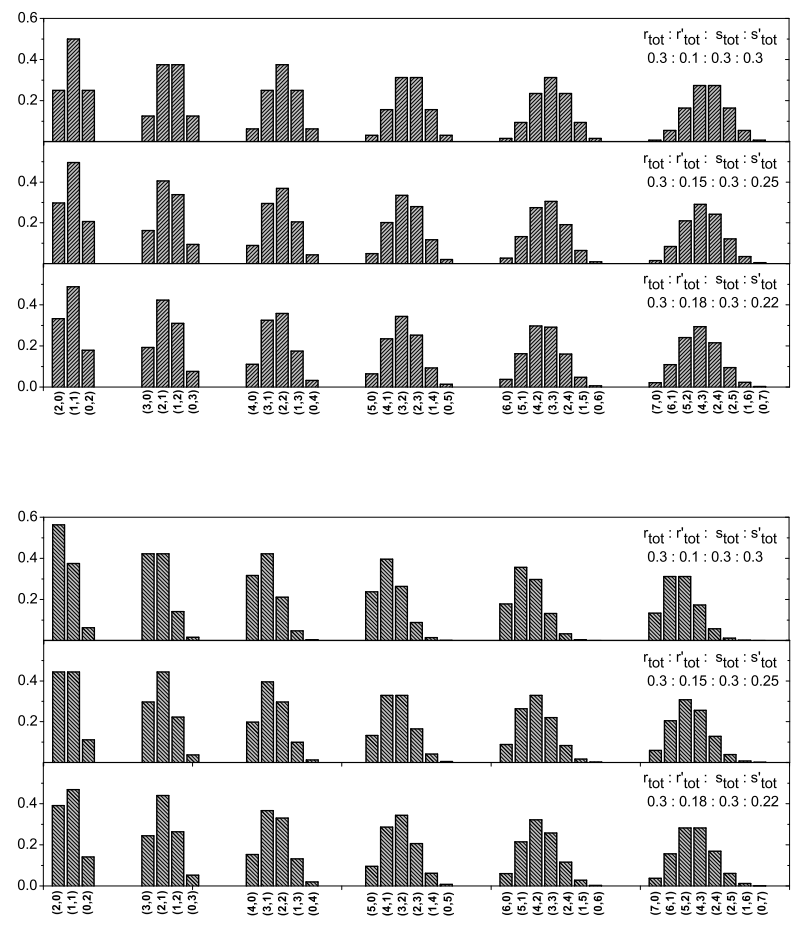

FIG. 8: Top set: mole fractions of the S-type copolymers $(n, m)$ for $m+n=N$ with $2 \leq N \leq 7$ (left to right in each row), as functions of the total initial fractions $r_{t o t}: r_{t o t}^{\prime}: s_{t o t}: s_{\text {tot }}^{\prime}$ indicated there. Bottom set: mole fractions of the R-type copolymers $(n, m)$ for $m+n=N$ with $2 \leq N \leq 7$. Since $s_{t o t}: s_{t o t}^{\prime}=1: 1$ in the first row of top graph, the distributions are binomial, but are skewed in all the other cases displayed.

TABLE IV: Average chain lengths for the two different starting compositions as a function of $K_{0}$ for $K_{1}=K_{0} / 2$ and $c_{t o t}=10^{-3} M$

\begin{tabular}{|c|c|c|c|c|c|c|c|c|c|c|c|c|c|c|}
\hline \multirow[b]{2}{*}{$K_{0}\left(M^{-1}\right)$} & \multicolumn{8}{|c|}{$r_{t o t}: r_{t o t}^{\prime}: s_{t o t}: s_{t o t}^{\prime}=0.3: 0.1: 0.3: 0.3$} & \multicolumn{6}{|c|}{$r_{\text {tot }}: r_{\text {tot }}^{\prime}: s_{\text {tot }}: s_{\text {tot }}^{\prime}=0.3: 0.14: 0.3: 0.26$} \\
\hline & $<l>$ & $<l_{S}>$ & $<l_{R}>$ & $<l_{S}^{s}>$ & $<l_{S}^{s^{\prime}}>$ & $<l_{R}^{r}>$ & $<l_{R}^{r^{\prime}}>$ & $<l>$ & $<l_{S}>$ & $<l_{R}>$ & $<l_{S}^{s}>$ & $<l_{S}^{s^{\prime}}>$ & $<l_{R}^{r}>$ & $<l_{R}^{r^{\prime}}>$ \\
\hline 1 & 2.00 & 2.00 & 2.00 & 2.00 & 2.00 & 2.00 & 2.00 & 2.00 & 2.00 & 2.00 & 2.00 & 2.00 & 2.00 & 2.00 \\
\hline 10 & 2.00 & 2.00 & 2.00 & 2.00 & 2.00 & 2.00 & 2.00 & 2.00 & 2.00 & 2.00 & 2.00 & 2.00 & 2.00 & 2.00 \\
\hline 100 & 2.04 & 2.04 & 2.03 & 2.03 & 2.01 & 2.03 & 2.00 & 2.04 & 2.04 & 2.04 & 2.03 & 2.01 & 2.03 & 2.00 \\
\hline 1000 & 2.33 & 2.36 & 2.28 & 2.19 & 2.12 & 2.22 & 2.04 & 2.33 & 2.35 & 2.30 & 2.20 & 2.10 & 2.22 & 2.05 \\
\hline 10000 & 3.77 & 3.94 & 3.53 & 2.53 & 2.45 & 2.88 & 2.16 & 3.75 & 3.86 & 3.61 & 2.58 & 2.40 & 2.78 & 2.22 \\
\hline 100000 & 8.62 & 9.23 & 7.83 & 2.80 & 2.77 & 3.81 & 2.27 & 8.58 & 8.79 & 8.13 & 2.89 & 2.67 & 3.44 & 2.37 \\
\hline
\end{tabular}

activation energies and may be present in solution in different proportions.

To resolve this problem, we must pay special attention to both the overall composition of the copolymer chain as well as its specific sequence. The problem has two basic parts: one is concerned with calculating the probability that a given amino-acid sequence is formed from the majority species and whatever minority species are present in their respective mole-fractions in solution. The attachment probability (the probability that the host template occludes this monomer) of an amino acid monomer to the growing chain is proportional to its concentration in solution and to a factor depending on its polymerization activation energy. The second part is to count the number of rearrangements or "shufflings" of the given sequence, as all these independent sequences will have the same probability to form as the given one. The information from both these parts will permit us to calculate the joint probability that a given sequence and its mirror image sequence are formed. This in turn will be used to provide a statistical measure of the likelihood that mirror symmetry is broken: below we derive a compact expression for the probability to find non-enantiomeric pairs of copolymers in the template (Figure 2). We first need to specify the length $N$ of the homochiral copolymer chains to be formed, and the number of each minority species or additive $m_{r}, m_{s}$. Thus we consider $\left(r_{0}, r_{1}, r_{2}, \ldots, r_{m_{r}}\right)$ 
TABLE V: Average chain lengths for the two different starting compositions as a function of $c_{t o t}$ for $K_{0}=100000$ and $K_{1}=K_{0} / 2$

\begin{tabular}{|c|c|c|c|c|c|c|c|c|c|c|c|c|c|c|}
\hline \multirow[b]{2}{*}{$c_{t o t}(M)$} & \multicolumn{8}{|c|}{$r_{\text {tot }}: r_{\text {tot }}^{\prime}: s_{\text {tot }}: s_{\text {tot }}^{\prime}=0.3: 0.1: 0.3: 0.3$} & \multicolumn{6}{|c|}{$r_{\text {tot }}: r_{\text {tot }}^{\prime}: s_{\text {tot }}: s_{\text {tot }}^{\prime}=0.3: 0.14: 0.3: 0.26$} \\
\hline & $<l>$ & $<l_{S}>$ & $<l_{R}>$ & $<l_{S}^{s}>$ & $<l_{S}^{s^{\prime}}>$ & $<l_{R}^{r}>$ & $<l_{R}^{r^{\prime}}>$ & $<l>$ & $<l_{S}>$ & $<l_{R}>$ & $<l_{S}^{s}>$ & $<l_{S}^{s^{\prime}}>$ & $<l_{R}^{r}>$ & $<l_{R}^{r^{\prime}}>$ \\
\hline $10^{-5}$ & 2.33 & 2.36 & 2.28 & 2.19 & 2.12 & 2.22 & 2.04 & 2.33 & 2.35 & 2.30 & 2.20 & 2.10 & 2.22 & 2.05 \\
\hline $10^{-4}$ & 3.77 & 3.94 & 3.53 & 2.53 & 2.45 & 2.88 & 2.16 & 3.75 & 3.86 & 3.61 & 2.58 & 2.40 & 2.78 & 2.22 \\
\hline $10^{-3}$ & 8.62 & 9.23 & 7.83 & 2.79 & 2.77 & 3.81 & 2.26 & 8.58 & 8.97 & 8.13 & 2.89 & 2.67 & 3.44 & 2.37 \\
\hline $10^{-2}$ & 23.99 & 25.99 & 21.50 & 2.92 & 2.92 & 4.49 & 2.31 & 23.90 & 25.16 & 22.48 & 3.06 & 2.80 & 3.86 & 2.43 \\
\hline $10^{-1}$ & 72.58 & 78.96 & 64.75 & 2.97 & 2.97 & 4.82 & 2.33 & 72.34 & 76.33 & 67.83 & 3.12 & 2.85 & 4.05 & 2.46 \\
\hline
\end{tabular}

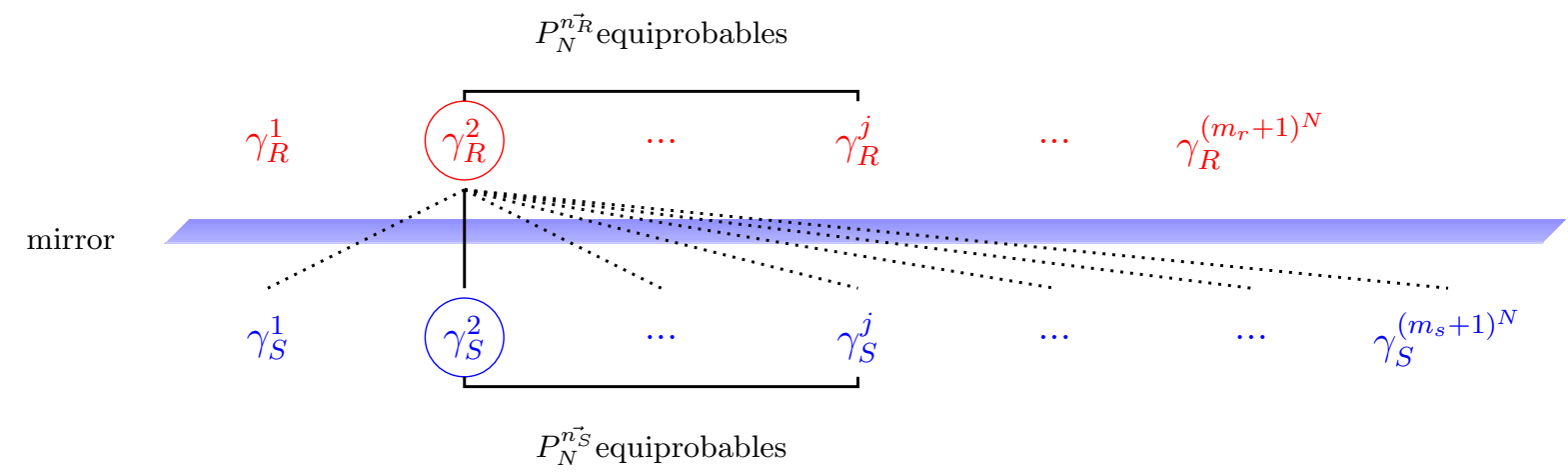

FIG. 9: Homochiral copolymer sequences and their mirror-related sequences. Top row (above the mirror) enumerates all the possible R-type copolymers $\gamma_{R}$ of length $N$ that can be made up from $m_{r}$ different R-type monomers: there are $\left(m_{r}+1\right)^{N}$ such chains. Below the mirror: mirror image related S-type homochiral copolymers $\gamma_{S}$ made up of S-type monomers. In this example $m_{r}<m_{s}$, so there are more S-type copolymers than R-type. Solid vertical line segment links an enantiomeric pair of sequences, the dotted lines represent examples of non-enantiomeric pairs of sequences. A given composition typically gives rise to many inequivalent but equiprobable sequences (indicated by the horizontal solid brackets).

and $\left(s_{0}, s_{1}, s_{2}, \ldots, s_{m_{s}}\right)$ whereas $(r, s) \equiv\left(r_{0}, s_{0}\right)$ denotes both the enantiomers of the majority species. Following the experimental scenario, the minority species will typically be present in small mole fractions, whereas the majority species will be present with a predominantly large mole fraction, as their names suggest. The total number of possible sequences in a chain with $N$ repeat units for each configuration is $\left(m_{r}+1\right)^{N}$ and $\left(m_{s}+1\right)^{N}$. This most general case is represented in a suggestive pictorial way in Fig. 9. This diagram is used to enumerate all possible chiral copolymers that can form in the template, laid out in a linear fashion, the totality of $R$-copolymers strung out above a "mirror" and the mirror-related $S$-copolymers directly below it.

Statistical copolymers are those for which the sequence of monomer residues follows a statistical rule. The attachment probability is proportional to the monomer's concentration in solution $\left[r_{j}\right],\left[s_{j}\right]$ times a rate constant that depends on the activation energy $E_{j}$ for attachment of that specific monomer to the polymer/template thus:

$$
\begin{aligned}
& p\left(r_{j}\right) \propto A_{j} \exp \left(-E_{j} / k T\right)\left[r_{j}\right]=w_{j}\left[r_{j}\right], \\
& p\left(s_{j}\right) \propto w_{j}\left[s_{j}\right] .
\end{aligned}
$$

To obtain bona-fide probabilities, these are are normalized so that

$$
\begin{aligned}
& 0 \leq p\left(r_{j}\right)=\frac{w_{j}\left[r_{j}\right]}{\sum_{k=0}^{m_{r}} w_{k}\left[r_{k}\right]} \leq 1,\left(0 \leq j \leq m_{r}\right) \\
& 0 \leq p\left(s_{j}\right)=\frac{w_{j}\left[s_{j}\right]}{\sum_{k=0}^{m_{s}} w_{k}\left[s_{k}\right]} \leq 1,\left(0 \leq j \leq m_{s}\right)
\end{aligned}
$$

which implies

$$
\sum_{k=0}^{m_{r}} p\left(r_{k}\right)=1, \quad \sum_{k=0}^{m_{s}} p\left(s_{k}\right)=1 .
$$


Normalization ensures that the probability that any single monomer attaches to the template is between zero and unity: evidently no individual probability can be greater than one, nor can the total probability exceed unity. In writing down Eq. (12), there are two implicit assumptions being made: (1) the rate of polymerization is independent of polymer length $N$, and (2), the probability of any given monomer joining a polymer is independent of the existing polymer structure. In (1) we are assuming isodesmic polymerization: the successive addition of a monomer to the growing chain leads to a constant decrease in the free energy, this in turn indicates that the affinity of a subunit for a polymer end is independent of the length of the polymer [18]. In (2) we assume the polymerization is a first-order Markov process, the attachment depends only on the nature of the terminal end of the polymer, but not on the monomer sequence in the chain. Evidence for kinetic Markov mechanisms has been observed experimentally in some chiral polymerizations [19].

Define the attachment probability vectors as

$$
\begin{aligned}
& \overrightarrow{p_{R}}=\left\{p\left(r_{0}\right), p\left(r_{1}\right), p\left(r_{2}\right), \ldots, p\left(r_{m_{r}}\right)\right\} \\
& \overrightarrow{p_{S}}=\left\{p\left(s_{0}\right), p\left(s_{1}\right), p\left(s_{2}\right), \ldots, p\left(s_{m_{s}}\right)\right\},
\end{aligned}
$$

one for the $R$-monomers, and one for the $S$-monomers. Note that in the limit when both minority species are absent $m_{r}, m_{s} \rightarrow 0$, there will be one unique sequence for each handedness. Namely, a sequence of $N R$ 's and a mirror sequence of $N S$ 's. These two pure sequences will each form with unit probability, since $p\left(r_{0}\right)=p\left(s_{0}\right)=1$, as follows from Eqs.(16). An enantiomeric pair will form with absolute certainty when there are no guest additives. This limit provides an important check on the statistical arguments developed below.

Chain compositions for the $R$ and $S$ type chains are specified as

$$
n\left(r_{0}\right)+n\left(r_{1}\right)+n\left(r_{2}\right)+\ldots+n\left(r_{m r}\right)=N,
$$

and

$$
n\left(s_{0}\right)+n\left(s_{1}\right)+n\left(s_{2}\right)+\ldots+n\left(s_{m_{s}}\right)=N,
$$

where $n\left(r_{j}\right)$ and $n\left(s_{j}\right)$ denote the number of times the $j$-th $R$ and $S$ monomer occur in the corresponding chain, respectively. These are ordered partitions of the integer $N$. Many different sequences can follow from one given composition Eqs. (19|20). By means of the template controlled polymerization mechanism [21], only homochiral chains will be formed, that means chains formed of either all right-handed $R$ or else all left-handed $S$ monomers, and these can be represented by vectors. For example, for the case of a right-handed chain:

$$
\gamma_{R}=\left\{r, r, r_{1}, r, r, r_{3}, \ldots ., r\right\}_{N},
$$

while its mirror image related sequence is denoted by the vector

$$
\gamma_{S}=\left\{s, s, s_{1}, s, s, s_{3}, \ldots, s\right\}_{N} .
$$

We emphasize that we are comparing the sequences of copolymers made up exclusively of either all right- $R$ or all left-handed $S$ monomers, and not making any claim about their corresponding secondary or terciary structures. When we discuss copolymers related through the mirror as in Fig. 9, we refer exclusively to their specific monomeric sequences or primary structures. It enumerates all the possible sequences that can form from the given composition. The underlying template control is assumed implicitly, thus the system is composed of only homochiral structures, see Figure 2 ,

The probability to form specific sequences of length $N$ from the compositions (19)20) is given by the composition probability:

$$
\begin{aligned}
& p\left(\boldsymbol{\gamma}_{R}\right)=\prod_{j=0}^{m_{r}} p\left(r_{j}\right)^{n\left(r_{j}\right)}, \\
& p\left(\boldsymbol{\gamma}_{S}\right)=\prod_{j=0}^{m_{s}} p\left(s_{j}\right)^{n\left(s_{j}\right)} .
\end{aligned}
$$

In general, there will be many distinct sequences with exactly the same composition-probability Eqs.(23|24), see the horizontal solid line segments in Figure 9. These are re-shufflings or re-orderings of the given sequence, keeping the individual composition numbers $n\left(r_{j}\right), n\left(s_{j}\right)$ fixed in (1920), and the number of such equiprobable sequences will be calculated below. 


\section{Probability to form one enantiomeric pair}

First consider the probability to form a specific sequence, call it $\gamma_{R}$. We fix the number of repeat units $N$ and the number $m_{r}$ of $R$-type additives. From the sequence we immediately deduce the composition (or composition vector) $\overrightarrow{n_{R}}=\left\{n\left(r_{0}\right), n\left(r_{1}\right), \ldots, n\left(r_{m_{r}}\right)\right\}$ and we specify the monomer attachment probabilities (or the attachment/occlusion probability vector), Eq.(17). Next, consider the probability to form its mirror image, that is, $\gamma_{S}$. The number of repeat units $N$ has been already fixed and we know the number $m_{s}$ of additives of $S$ type. The composition vector of the mirror image $\overrightarrow{n_{S}}$ must be equal to $\overrightarrow{n_{R}}$, that is $\overrightarrow{n_{S}}=\overrightarrow{n_{R}} \equiv \vec{n}$, and we must specify the monomer attachment probabilities (or the attachment/occlusion probability vector), Eq.(18).

The probabilities to form these sequences from these compositions are given by Eq.(23) 24) and hence the joint probability to find the enantiomeric pair $\gamma_{R}$ and $\gamma_{S}$ is

$$
P_{\text {pair }}\left(\gamma_{R} \mid \gamma_{S}\right)=p\left(\gamma_{R}\right) p\left(\gamma_{S}\right)
$$

This is a function of $N, \min \left(m_{r}, m_{s}\right), \vec{n}, \overrightarrow{p_{R}}$ and $\overrightarrow{p_{S}}$.

\section{Probability to form all possible enantiomeric pairs for fixed $N$}

For computing the probability of forming all possible enantiomeric pairs, we need to know both $m_{r}$ and $m_{s}$ and which one is greater, since limits on the possible enantiomeric pairs that can be formed come from the enantiomer with the least number of guest species. Without loss of generality we may assume that $m_{r} \leq m_{s}$. For fixed $N$ and $m_{r}$, the number of distinct compositions of the $R$ type copolymers is given by

$$
\#_{N, m_{r},\left\{n_{0}, n_{1}, n_{2}, \ldots n_{m_{r}}\right\}}=\left(\begin{array}{c}
m_{r}+N \\
N
\end{array}\right)=\frac{\left(m_{r}+N\right) !}{N ! m_{r} !}
$$

and the number of different sequences that we can form from each individual composition is given by

$$
P_{N}^{\overrightarrow{n_{R}}} \equiv P_{N}^{n_{0} n_{1} n_{2} \ldots n_{m_{r}}}=\left(\begin{array}{c}
N \\
n_{0}, n_{1}, n_{2}, \ldots, n_{m_{r}}
\end{array}\right)=\frac{N !}{n_{0} ! n_{1} ! n_{2} ! \ldots n_{m_{r}} !} .
$$

Summing the latter expression over all the possible compositions with fixed $N$ must be equal to the total number of different sequences, that is, we obtain the multinomial theorem [30]: $\sum\left(\begin{array}{c}N \\ n_{0}, n_{1}, n_{2}, \ldots, n_{m_{r}}\end{array}\right)=\left(m_{r}+1\right)^{N}$.

Recall, the joint probability to form a particular sequence and its mirror image sequence is given by Eq. (25). The net probability we seek to evaluate is:

$$
P_{\text {pairs }}\left(N, m_{r}\right)=\sum_{\gamma_{R}} P_{\text {pair }}\left(\gamma_{R} \mid \gamma_{S}\right)
$$

This expression is the probability that each and every possible sequence in $R$ and its mirror image sequence in $S$ are formed of fixed length $N$. For this purpose, we will first sum over all different (but equiprobable) sequences belonging to the same composition and then, sum over all different compositions for $N$ repeat units. That is $\sum_{a l l-s e q u e n c e s}=$ $\sum_{\text {compositions }}\left(\sum_{\text {equiprobable-sequences }}\right)$. From Eq. (27) each given composition can be rearranged in $P_{N}^{\vec{n}}$ different ways. For a given composition, all the sequences that can be made therefrom (re-shufflings) are equiprobable. Thus summing over all these possible rearrangements, we arrive at the probability to form chains and their mirror image sequences within one such equiprobable equivalence class, recall $m_{r}<m_{s}$ :

$$
P_{N}^{\overrightarrow{n_{R}}} p\left(\gamma_{R}\right) p\left(\gamma_{S}\right)=\frac{N !}{n_{0} ! n_{1} ! n_{2} ! \ldots n_{m_{r}} !} \prod_{j=0}^{m_{r}} p\left(r_{j}\right)^{n\left(r_{j}\right)} \prod_{j=0}^{m_{r}} p\left(s_{j}\right)^{n\left(s_{j}\right)} .
$$

Finally, summing this result over all the different compositions, we calculate the net probability to form homochiral chains and their mirror image sequences in the system: i.e., the probability to form all possible enantiomeric pairs. Thus, the probability that mirror symmetry is not broken for $m$ additives and $N$ repeat units is given by

$$
\begin{aligned}
P_{\text {nobreak }}\left(N, m_{r}\right) & =P_{\text {pairs }}\left(N, m_{r}\right) \\
& \sum_{n_{0}+n_{1}+n_{2}+\ldots+n_{m_{r}}=N} P_{N}^{\vec{n}} p\left(\gamma_{R}\right) p\left(\gamma_{S}\right) .
\end{aligned}
$$


Then the probability that mirror symmetry is broken for these values of $m$ and $N$ is:

$$
\begin{aligned}
P_{\text {break }}\left(N, m_{r}\right)= & P_{\text {no pairs }}\left(N, m_{r}\right) \\
= & \sum_{n_{0}+n_{1}+n_{2}+\ldots+n_{m_{r}}=N} P_{N}^{\overrightarrow{n_{R}}} p\left(\gamma_{R}\right)\left(1-p\left(\gamma_{S}\right)\right) \\
= & 1-\sum_{n_{0}+n_{1}+n_{2}+\ldots+n_{m_{r}}=N} P_{N}^{\overrightarrow{n_{R}}} p\left(\gamma_{R}\right) p\left(\gamma_{S}\right) \\
= & 1-\left(p_{r_{0}} p_{s_{0}}+p_{r_{1}} p_{s_{1}}+\ldots+p_{r_{m_{r}}} p_{s_{m_{r}}}\right)^{N}, \\
= & 1-\left(\vec{p}_{R} \cdot \vec{p}_{S}\right)^{N} .
\end{aligned}
$$

which follows from the multinomial theorem, [30] and after using Eqs.(16/17/18).

\section{B. Chiral additives}

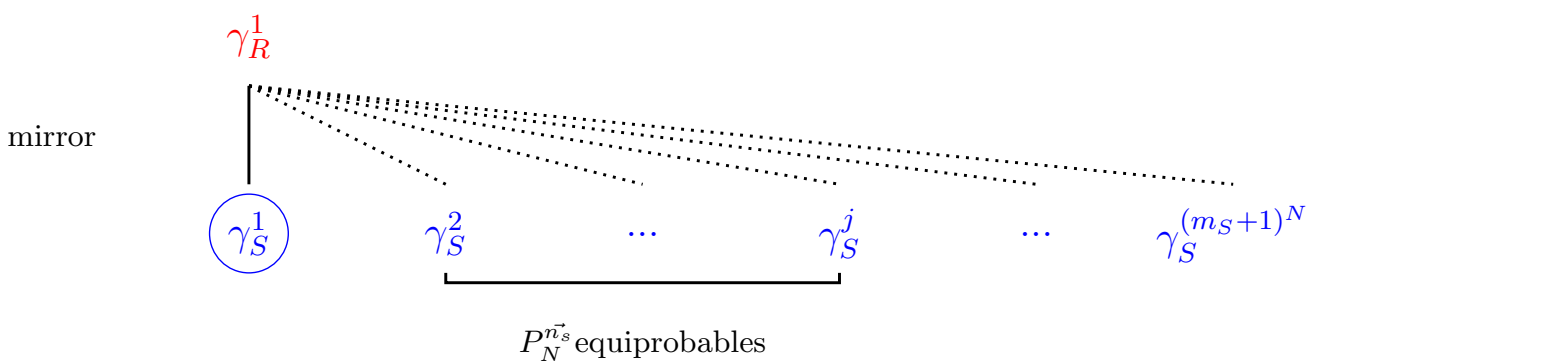

FIG. 10: Pictorial situation for chiral guest additives. In this case, only additives of one chirality (in $S$ ) are added. So $m_{r}=0$ and $m_{s}=m$; with $N$ fixed. Top row (above the mirror) the unique R-type polymer of length $N$ that can be made up from the single R-type monomer present in the system. Below the mirror: all the S-type copolymers made up of $m$ distinct S-type monomers. Solid vertical line indicates the single unique enantiomeric pair, the dotted lines represents all the non-enantiomeric pairs of sequences for $m_{s} \geq 1$

This is a special case of the general one described above, and is pictorially sketched out in Fig. 10 Here we consider $m_{r}=0$ and we set $m_{s}=m$. Clearly, there is only one possible composition (and hence, sequence) that can be formed in $R$, namely the pure homochiral sequence made up of $N$ repeat units of $r$ : namely $\gamma_{R}=\{r, r, \ldots, r\}_{N}$, and this forms with unit probability: $p\left(\gamma_{R}\right)=1$. Thus, the probability that mirror symmetry is not broken for $m$ types of $S$-additives and for $N$ repeat units is given by Eq.(30), which simplifies to give:

$$
\begin{aligned}
P_{\text {nobreak }}(N, m) & =P_{\text {pairs }}(N, m) \\
& =p\left(\gamma_{R}\right) p\left(\gamma_{S}\right)=p\left(s_{0}\right)^{N} .
\end{aligned}
$$

Then the probability that mirror symmetry is broken for these values of $m$ and $N$ is:

$$
\begin{aligned}
P_{\text {break }}(N, m) & =P_{\text {no pairs }}(N, m) \\
& =p\left(\gamma_{R}\right)\left(1-p\left(\gamma_{S}\right)\right) \\
& =1-p\left(s_{0}\right)^{N} .
\end{aligned}
$$

If the number of $S$-type additives goes to zero, $m_{s} \rightarrow 0$, then $p\left(s_{0}\right) \rightarrow 1$ and then mirror symmetry is maintained with absolute certainty.

\section{Ideal Racemic Additives}

For our final example, we deal with the case in which all the additives are supplied in ideally racemic proportions, that is, we have equal numbers of enantiomer types $m_{r}=m_{s} \equiv m$, and all are supplied in identical concentrations: 


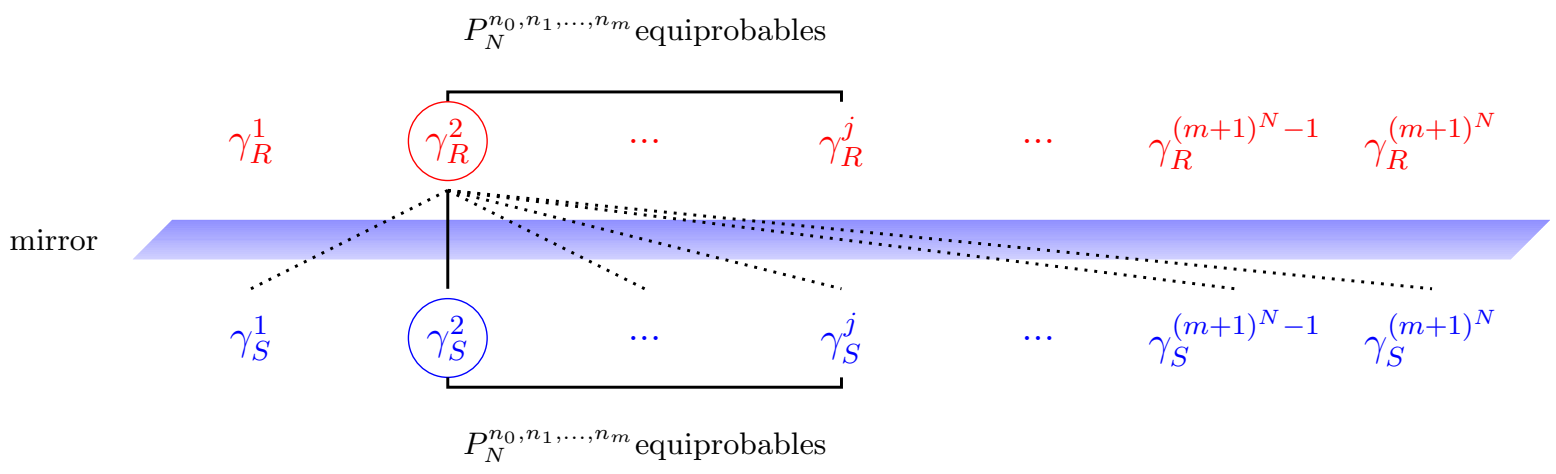

FIG. 11: Pictorial scheme for the case of all racemic additives: $m_{r}=m_{s}=m ; N$ is fixed. Compare to Figure 9 .

$\left[r_{j}\right]=\left[s_{j}\right]$ for all types $0 \leq j \leq m=m_{r}=m_{s}$. This situation is pictorially represented in Fig. 11, Since the activation energies are the same for both $R$ and $S$ enantiomers, then from Eqs. (14]15) $p\left(r_{j}\right)=p\left(s_{j}\right)$ and so from Eqs. (23|24) $p\left(\gamma_{R}\right)=p\left(\gamma_{S}\right) \equiv p(\gamma)$. All the sequences that are obtained from re-shuffling the original one have the same probability for polymerizing: they define equivalence classes of equiprobable sequences. This number gives the number of distinct equivalence classes of equiprobable sequences (Figure 11). An important check on the formalism and numerics that follow from it is that the probability for mirror symmetry breaking must go to zero as the number $m$ of (ideally racemic) monomer additives goes to zero. We will see that these expectations are confirmed.

For ideally racemic additives, the probability that mirror symmetry is not broken for $m$ additives and $N$ repeat units is given by Eq.(30), which reduces to:

$$
P_{\text {nobreak }}(N, m)=\left(\left(\vec{p}_{R}\right)^{2}\right)^{N} .
$$

Now, from Eq. (31) the probability that mirror symmetry is broken for $m$ species of racemic additives and for chain length $N$ is

$$
P_{\text {break }}(N, m)=1-\left(\left(\vec{p}_{R}\right)^{2}\right)^{N} .
$$

This result is important: it says that even for ideally racemic initial proportions in all the host and guest amino acids, there is a finite probability $1 \geq P_{b r e a k}(N, m)>0$ for statistical or stochastic breaking of mirror symmetry. This mirror symmetry breaking is manifested in the formation of non-enantiomeric pairs of homochiral sequences within the template, in support of the proposed experimental scenario [21].

\section{RESULTS}

From Eqs. (14 [18) the monomer attachment probability vectors $\vec{p}_{R}$ and $\vec{p}_{S}$ define the faces of two standard or unit $m_{r}$ and $m_{s}$-simplexes [31]. These simplex faces represent the domains of all allowed monomer attachment probabilities, see the shaded regions in Fig. 12 and Fig. 13. This allows us to find the basic physico-chemical criteria for maximizing (or minimizing) the probability for broken mirror symmetry in template-controlled polymerization 21, 22, 25]. For an $m$-simplex there is a maximum and a minimum distance from the origin. The maximum distance pertains when the attachment probability vector $\vec{p}$ coincides with one of the $m+1$ vertices, and in these cases we have $\|\vec{p}\|=1$. The minimum distance is achieved for the point defined by the centroid of the simplex face located at $\vec{p}=\left\{\frac{1}{m+1}, \frac{1}{m+1}, \ldots ., \frac{1}{m+1}\right\}$ (a vector with $(m+1)$-components) and its modulus is $\|\vec{p}\|=1 / \sqrt{m+1}$.

\section{A. General case: non-racemic additives.}

For this general case in which the number of additives of each enantiomer type can be distinct, the attachment probability vectors $\vec{p}_{R}$ and $\vec{p}_{S}$ have $m_{r}$ and $m_{s}$ components, respectively. In principle, they are vectors in simplexes of different dimensions. The limits on the number of possible mirror related copolymer pairs that can be formed come from the enantiomer with the least number of additives, which, without loss of generality, we take to be $m_{r}$. 


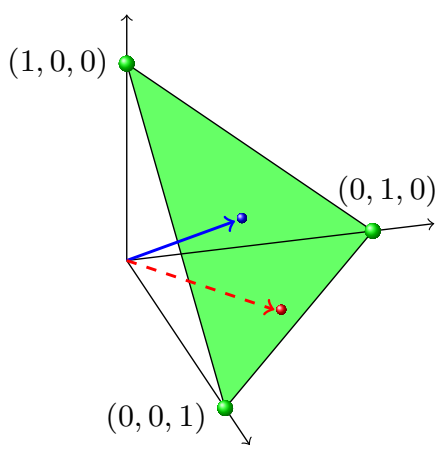

FIG. 12: The unit $m$-simplex, illustrated for the case of $m=2$ amino acid additives. The three vertices are located at the points $(1,0,0),(0,1,0)$ and $(0,0,1)$ and correspond to maximum attachment probabilities (mirror symmetry is conserved). Shaded area (green) corresponds to the domain of all allowed attachment vectors. A generic point (broken arrow) corresponds to a positive probability for symmetry breaking. The centroid $(1 / 3,1 / 3,1 / 3)$ (solid arrow), corresponds to the maximum probability for mirror symmetry breaking.

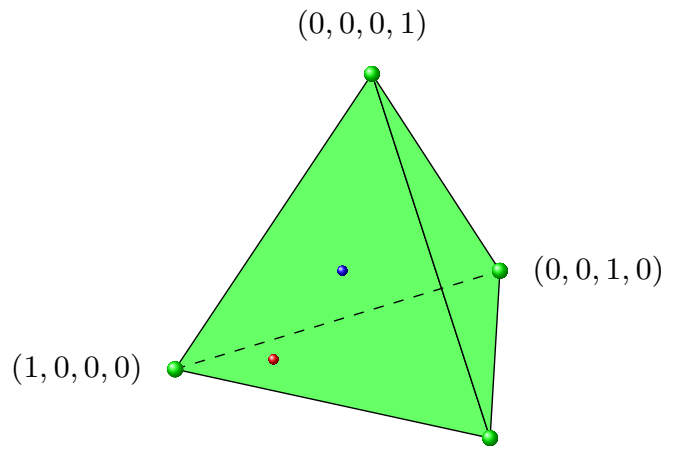

$(0,1,0,0)$

FIG. 13: The unit $m$-simplex, illustrated for the case of $m=3$ additives. The four vertices are located at the points $(1,0,0,0),(0,1,0,0),(0,0,1,0)$ and $(0,0,0,1)$ and correspond to maximum attachment probabilities (mirror symmetry is conserved). The centroid (blue dot) corresponds to maximal mirror symmetry breaking.

Express the vector $\vec{p}_{R}$ in the $m_{s}$ simplex, by simply redefining $\vec{p}_{R}=\left\{p_{r_{0}}, p_{r_{1}}, \ldots, p_{r_{m_{r}}}, 0,0, . ., 0\right\}$ with $m_{s}-m_{r}$ zero entries, then the probability for mirror symmetry breaking follows from Eq. 31

$$
P_{b r e a k}\left(N, m_{r}\right)=1-\left(\vec{p}_{R} \cdot \vec{p}_{S}\right)^{N} \text {. }
$$

Now, both attachment vectors can be regarded as belonging to the same $m_{s}$-simplex. This can be visualized graphically as a $m_{s}$-simplex with one of its subspaces being the $m_{r}$-simplex (the subspace can be just a point of the $m_{s^{-}}$ simplex $\left(m_{r}=0\right)$, a line $\left(m_{r}=1\right)$, a face $\left(m_{r}=2\right)$, etc). Fig. 14 represents the case in which $m_{s}=3$ and $m_{r}=2$, here the subspace of the $m_{s}$-simplex corresponding to the $m_{r}$-simplex is a face of the tetrahedron. Then the probability of breaking symmetry is minimal (zero) when the attachment probability vectors $\vec{p}_{R}$ and $\vec{p}_{S}$ are parallel, for then $\vec{p}_{R} \cdot \vec{p}_{S}=1$ and $P_{\text {break }}\left(N, m_{r}\right)=0$. In order for both vectors to be parallel, both must be in the subspace of the $m_{s}$-simplex that coincides with the $m_{r}$-simplex. The maximum probability for breaking mirror symmetry $P_{\text {break }}\left(N, m_{r}\right)=1$ is achieved when the attachment probability vectors are orthogonal and thus coinciding with two different vertices of the $m_{s}$-simplex (see Figure 13). $\vec{p}_{R}$ must be in one of the $m_{r}+1$ vertices and $\vec{p}_{S}$ can be in one of the $m_{s}+1$ vertices, always different from the vertex in which $\vec{p}_{R}$ lies. In this case, the species in $r$ will be different from the species in $s$, so it will be impossible to form enantiomeric pairs of homochiral chains.

Typically, copolymers will be formed with lengths ranging from the dimer, trimer, etc. on up to a maximum number of repeat units $N[21,22,25]$. The above arguments apply to any value of $N$, thus the probability $P_{b r e a k}^{\leq N}(m)$ to break 
mirror symmetry in a system containing a spectrum of chain lengths $2 \leq n \leq N$ is given as follows,

$$
\begin{aligned}
P_{\text {break }}^{\leq N}\left(m_{r}\right) & =\frac{1}{N-1} \sum_{n=2}^{N} P_{\text {break }}\left(n, m_{r}\right) \\
& =1-\frac{\left(\vec{p}_{R} \cdot \vec{p}_{S}\right)^{2}\left(1-\left(\vec{p}_{R} \cdot \vec{p}_{S}\right)^{N-1}\right)}{(N-1)\left(1-\vec{p}_{R} \cdot \vec{p}_{S}\right)},
\end{aligned}
$$

and satisfies $\lim _{\vec{p}_{R} \cdot \vec{p}_{S} \rightarrow 1} P_{\text {break }}^{\leq N}(m)=0$ when the two occlusion probability vectors are parallel.

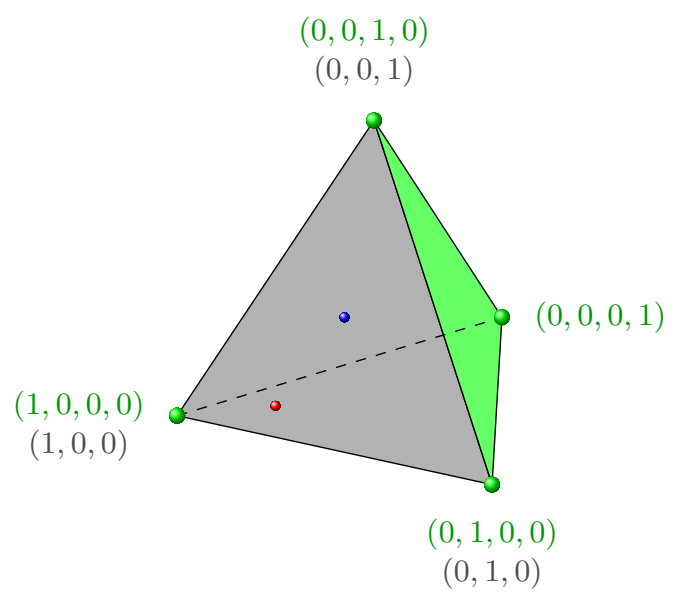

FIG. 14: Both the unit $m_{r}$ and $m_{s}$-simplexes, illustrated for the particular case of $m_{r}=2$ and $m_{s}=3$ additives. The three vertices of the $m_{r}$-simplex are located at the points $(1,0,0),(0,1,0)$ and $(0,0,1)$ and coincide to maximum attachment probabilities of $r$. These three vertices of the $m_{r}$-simplex also coincide with three of the four vertices of the $m_{s}$-simplex, located at the points $(1,0,0,0),(0,1,0,0),(0,0,1,0)$ and $(0,0,0,1)$ and corresponding to maximum attachment probabilities of $s$

\section{B. Additives of only one handedness.}

This is a particular case of the above for $m_{r} \neq m_{s}$, when $m_{r}=0$. The probability of breaking mirror symmetry depends only on $p\left(s_{0}\right)$ : the attachment probability of the $S$-enantiomer of the majority species. Each monomer attachment vector is in a different simplex with different dimensions, $\vec{p}_{S}$ is in a $m_{s}$-simplex and $\vec{p}_{R}$ will coincide with a vertex of the $m_{s}$-simplex.

The minimal probability of breaking symmetry $P_{\text {break }}(N, m)=0$ is obtained for $p\left(s_{0}\right)=1$, in this case, there are no guests, only the majority species $S_{0}$, so we recover the case in which additives are supplied in racemic proportions, and moreover, no guests are added. In this case, the attachment probability vector $\vec{p}_{S}$ coincides with one of the $m+1$ vertices, the vertex corresponding to $\vec{p}_{R}$ and to $p\left(s_{0}\right)$ maximum. The maximum probability of breaking mirror symmetry $P_{b r e a k}(N, m)=1$ is obtained for $p\left(s_{0}\right)=0$. In this case, the majority species in $S$, is absent, thus no possible enantiomeric pairs can be formed, that is, the vector $\vec{p}_{s}$ can lie anywhere in the $m_{s}$-simplex, except at the $m_{r}$ vertex.

In this case, the probability $P_{b r e a k}^{\leq N}(m)$ to break mirror symmetry in a system containing a spectrum of chain lengths $2 \leq n \leq N$ Eq. (37) reduces to

$$
P_{b r e a k}^{\leq N}(m)=1-\frac{p\left(s_{0}\right)^{2}\left(1-p\left(s_{0}\right)^{N-1}\right)}{(N-1)\left(1-p\left(s_{0}\right)\right)}
$$

and satisfies $\lim _{p\left(s_{0}\right) \rightarrow 1} P_{\text {break }}^{\leq N}(m)=0$ when no majority specie of the S-type is supplied.

The cases with two majority species $r$ and $s$ and one guest, $s^{\prime}$, with starting fractions $f_{r}: f_{s}: f_{s^{\prime}}$, as considered in the first section of the paper, would be a case of additives of only one handedness or chiral additive, where $m_{r}=0$ and $m_{s}=m=1$. Following Eq. (33) we can calculate $P_{b r e a k}^{\leq N}(m)$ for the three different starting compositions considered before. Exemplary numerical results are shown in the Tables (VI), (VII) and in Figure[15)showing the effect of varying the relative concentrations of all the monomers and the activation energy (we vary $w_{s}^{\prime}$ ) of the guest monomer $s^{\prime}$. The curves for $P_{b r e a k}$ are qualitatively similar to those of the percent $e e$ in Figure 3 . 
TABLE VI: $w_{r}=w_{s}=w_{s^{\prime}}=1$ Probability to break mirror symmetry, $P_{b r e a k}^{\leq N}(m)$, for the three different starting composition $f_{r}: f_{s}: f_{s^{\prime}}$ of the three component case $\left(m_{r}=0\right.$ and $\left.m_{s}=m=1\right)$ as a function of repeat units $N$

\begin{tabular}{lllllll}
\hline$P_{\text {break }}^{\leq N}(m)$ & $N=5$ & $N=10$ & $N=15$ & $N=20$ & $N=25$ & $N=30$ \\
$0.5: 0.25: 0.25$ & 0.88 & 0.94 & 0.96 & 0.97 & 0.98 & 0.98 \\
$0.5: 0.45: 0.05$ & 0.30 & 0.45 & 0.55 & 0.63 & 0.69 & 0.73 \\
$0.5: 0.475: 0.025$ & 0.16 & 0.26 & 0.34 & 0.41 & 0.47 \\
\hline
\end{tabular}

TABLE VII: $w_{r}=w_{s}=1, w_{s^{\prime}}=0.75$ Probability to break mirror symmetry, $P_{b r e a k}^{\leq N}(m)$, for the three different starting composition $f_{r}: f_{s}: f_{s^{\prime}}$ of the three component case $\left(m_{r}=0\right.$ and $\left.m_{s}=m=1\right)$ as a function of repeat units $N$

\begin{tabular}{lllllll}
\hline$P_{\text {break }}^{\leq N}(m)$ & $N=5$ & $N=10$ & $N=15$ & $N=20$ & $N=25$ & $N=30$ \\
\hline $0.5: 0.25: 0.25$ & 0.83 & 0.92 & 0.95 & 0.96 & 0.97 & 0.97 \\
$0.5: 0.45: 0.05$ & 0.24 & 0.37 & 0.47 & 0.54 & 0.61 & 0.66 \\
$0.5: 0.475: 0.025$ & 0.12 & 0.22 & 0.27 & 0.33 & 0.39 & 0.43 \\
\hline
\end{tabular}

\section{Racemic additives.}

When the enantiomeric species are provided in ideally racemic proportions, the probability that mirror symmetry is broken for given values of the chain length $N$ and number of species $m$ can be expressed succinctly as

$$
P_{\text {break }}(N, m)=1-\|\vec{p}\|^{2 N}
$$

that is, one minus the squared-modulus of the probability attachment vector $\vec{p}$, raised to the chain length. Thus, for fixed $N$, in order to maximize the probability that mirror symmetry be broken, we should prepare the chemical system so that all $m$ additives and the majority species have equally shared mole fractions. For any other point in the face (including the centroid), but excluding the $m+1$ vertices, then $\|\vec{p}\|<1$, hence the probability to break mirror symmetry increases with chain length $N$ and/or with increasing number of additives $m$, provided these are supplied with small mole fractions (to prevent $\vec{p}$ from coinciding with the vertices).

Finally, if the occlusion probability vector $\vec{p}$ coincides with any one of the $m+1$ vertices, then $\|\vec{p}\|=1$, so $P_{\text {break }}(N, m)=0$, and mirror symmetry is maintained with absolute certainty for all $N$. Each vertex corresponds to a chemical system with only one type of monomer (and its enantiomer), in other words, a system with no additives. The $m$-th vertex corresponds to the $m$-th amino acid being the sole species present in the system. Thus we see that if we increase the mole fraction of any one of the additives in excess, the tables are turned, and the majority and minority species interchange their roles: excessive amounts of any additive tend to reduce the probability for breaking mirror symmetry.

Eq. (37) now simplifies to give

$$
P_{\text {break }}^{\leq N}(m)=1-\frac{\|\vec{p}\|^{4}\left(1-\|\vec{p}\|^{2(N-1)}\right)}{(N-1)\left(1-\|\vec{p}\|^{2}\right)}
$$

and satisfies $\lim _{\|\vec{p}\| \rightarrow 1} P_{\text {break }}^{\leq N}(m)=0$ at the vertices of the simplex. As expected, we find increasing probability for symmetry breaking as $N$ and/or $m$ increase. A comparison of the two Tables confirms that the probabilities are maximized for each $N$ and $m$, when all species are supplied in equal proportions. The probability to break mirror symmetry is strictly zero when there are no additives: $P_{b r e a k}^{\leq N}(m=0)=0$.

The cases with two majority species $r$ and $s$ and two guests, $r^{\prime}$ and $s^{\prime}$, with starting fractions $f_{r}: f_{r^{\prime}}: f_{s}: f_{s^{\prime}}$, as considered in the first section of the paper, is a case of racemic additives where $m_{r}=m_{s}=1$. Following Eq.(35) we can calculate $P_{b r e a k}^{\leq N}(m)$ for the three different starting compositions considerer before. The results (not shown) are qualitatively very similar to to those shown in the previous tables.

\section{CONCLUSIONS}

The proposed 21] experimental mechanism leads to the formation of homochiral copolymers with random sequences of the majority and minority amino acids. Given the implications of the experimental mechanism, we have provided two independent and complementary theoretical approaches to the problem. The first one is based on approximate 


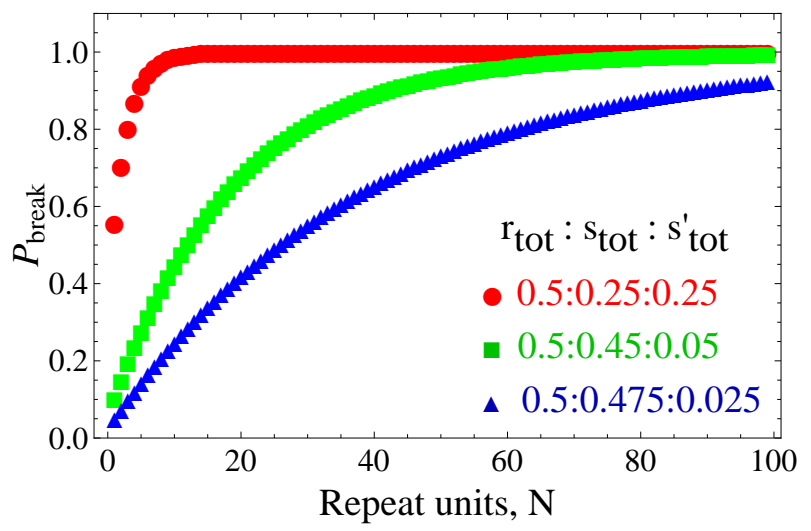

FIG. 15: $w_{r}=w_{s}=1, w_{s^{\prime}}=0.5$. Probability to break mirror symmetry, $P_{b r e a k}^{\leq N}(m)$, for the three different starting composition $f_{r}: f_{s}: f_{s^{\prime}}$ of the three component case $\left(m_{r}=0\right.$ and $\left.m_{s}=m=1\right)$ as a function of repeat units $N$.

chemical equilibrium, the second, on statistical principles and combinatorics. Both these approaches provide further quantitative insights into the template-controlled induced desymmetrization mechanisms advocated by Lahav and coworkers [20 26].

In the first approach, appealing to chemical equilibrium, the template or beta sheet is in approximate equilibrium with the free monomer pool. We obtain a multinomial sample space for the distribution of equilibrium concentrations of the homochiral copolymers. We then deduce mass balance equations for the enantiomers of the individual amino acid species, and their numerical solutions are used to evaluate the sequence-dependent copolymer concentrations, in terms of the total species concentrations. Measurable quantities signalling the degree of mirror symmetry breaking such as the enantiomeric excess $e e$, relative abundances and average chain lengths are evaluated as functions of initial monomer concentrations and the individual equilibrium constants. We can take these constants as large as desired to approximate irreversible polymerization.

The second, or probabilistic, description confirms that this is a viable mechanism for stochastic mirror symmetry breaking. We give criteria for the chemical conditions leading to either maximal or minimal probabilities for breaking mirror symmetry in this experimental context. The probability for finding non-enantiomeric pairs of copeptide chains of different sequences increases as a function of increasing chain length and increasing number of guest amino acids. We can calculate the probabilities of all these joint outcomes in terms of the basic monomer attachment/occlusion probabilities. These probabilities can be calculated in terms of the monomer concentrations and take into account the fact that different amino acids have different polymerization activation energies. The solution of the full problem admits an appealing visual and geometric interpretation in terms of unit-simplexes which summarize the allowed relative polymerization rates and concentrations of the different amino acids involved.

There are two important points worth emphasizing. First, our theoretical models invoke the underlying template control in that they do not allow for any heterochiral oligomers to form. The sequence of the host and guest amino acids within the homochiral peptides assembles in a completely random fashion, in accord with the experiments [21]. This sequence randomness is captured by both the model based on chemical equilibrium and by the second model based on the monomer occlusion probabilities. Secondly, the statistical/combinatorial effects do lead to a stochastic mirror symmetry breaking effect. The symmetry breaking in these experiments arises from combinatorics, not from spontaneous (bifurcation) phenomena. These stochastic/statistical/combinatorial effects are not due to the inherent tiny chiral fluctuations present in all real chemical systems 39, 40, 43] but are due rather to the random occlusion of host and guest amino acids by the chiral sites of the template: the mechanisms proposed here work even for ideally racemic mixtures. Mirror symmetry is broken in the sequences, as non-enantiomeric pairs of oligomers are formed. The solution of free monomers can nevertheless be optically inactive. The symmetry breaking is to be found in the template, or $\beta$-sheet, but not (necessarily) in the solution.

An important distinction must be drawn between the types of symmetry breaking/amplification treated in this paper. Whereas in the first part (Sec III) treats the global system symmetry (that thus can lead to global chiral effects), the one described in this latter part (Sec IV) concerns local asymmetries (specific all-R versus all-S amino acid sequences), that could be invisible at the global scale. It is not guaranteed that one asymmetry will imply the other.

The experiments 21] motivating the present study shed valuable light on the role of templates in the origin of homochiral peptides. Recent works have discussed the potential roles of peptide (amino acid) $\beta$-sheets in the origin of life, underscoring their effective protection against decomposition and racemization (recovery of mirror symmetry) as well as their catalytic ability towards hydrolysis [33, 34]. An experimental demonstration of the formation of $\beta$-sheets 
that serve as catalysts for peptide condensation and self-replication has been reported recently 35]. Other groups have demonstrated that $\beta$-sheet templates can affect enzyme assisted amino acid polymerization [36], and replication of cylic peptide structures [37]. Such templates might have enjoyed a considerable enantioselective advantage in a prebiotic environment [38].

In closing, we note that the symmetry breaking mechanism of Lahav and coworkers [21, 25] has some features in common with the qualitative scenarios of Green, Eschenmoser and Siegel in which a deficient or limited supply of material results in a stochastic symmetry breaking process 41 43].

\section{Acknowledgements}

We are grateful to Meir Lahav (Weizmann Institute of Science) for proposing this problem and for encouraging us to find a mathematical model for the template copolymerization mechanism, and for many useful discussions and correspondence. We also acknowledge Gonen Ashkenasy (Ben Gurion University of the Negev) for recent discussions about models for $\beta$-sheets. Comments from an anonymous reviewer have helped to improve the manuscript. CB has a Calvo Rodés predoctoral contract from the Instituto Nacional de Técnica Aeroespacial (INTA). DH acknowledges a grant AYA2009-13920-C02-01 from the Ministry of Science and Innovation (currently MINECO).

[1] Guijarro, A.; Yus, M. The Origin of Chirality in the Molecules of Life; RSC Publishing: Cambridge, 2009.

[2] Sandars, P.G.H. Origins Life Evol. Biosphere 2003, 33, 575-587.

[3] Frank, F.C. Biochim. Biophys. Acta 1953, 11, 459-463.

[4] Brandenburg, A.; Multamäki, T. Int. J. Astrobiol. 2004, 3, 209-219.

[5] Brandenburg, A.; Andersen, A.C.; Höfner, S.; Nilsson, M. Origins Life Evol. Biosphere 2005, 35, $225-241$.

[6] Wattis, J.A.D.; Coveney, P.V. Origins Life Evol. Biosphere 2005, 35, 243-273.

[7] Gleiser, M.; Thorarinson, J. Origins Life Evol. Biosphere 2006, 36, 501-505.

[8] Gleiser, M. Origins Life Evol. Biosphere 2007, 37, 235-251.

[9] Gleiser, M.; Walker, S.I. Origins Life Evol. Biosphere 2008, 38, 293-315.

[10] Gleiser, M.; Thorarinson, J.; Walker, S.I. Origins Life Evol. Biosphere 2008, 38, 499-508.

[11] Blocher, M.; Hitz, T.; Luisi, P.L. Helv. Chim. Acta 2001, 84, 842-848.

[12] Hitz, T.; Blocher, M.; Walde, P.; Luisi, P.L. Macromolecules 2001, 34, 2443-2449.

[13] Bada, J.L.; Miller, S.L. Biosystems 1987, 20, 21-26.

[14] Avetisov, V.A.; Goldanskii, V.I.; Kuzmin, V.V. Dokl. Akad. Nauk USSR 1985, 282, 115-117.

[15] Goldanskii, V.I.; Avetisov, V.A.; Kuzmin V.V. FEBS Lett. 1986, 207, 181-183.

[16] Avetisov, V.; Goldanskii, V. Proc. Natl. Acad. Sci. USA 1996, 93, 11435-11442.

[17] Orgel, L.E. Nature 1992, 358, 203-209.

[18] De Greef, T.F.A.; Smulders, M.M.J.; Wolffs, M.; Schenning, A.P.H.J.; Sijbesma, R.P.; Meijer, E.W. Chem. Rev. 2009, 109(11), 5687-5754.

[19] Weissbuch, I.; Illos, R.A.; Bolbach, G.; Lahav, M. Acc. Chem. Res. 2009, 42, 1128-1140.

[20] Zepik, H.; Shavit, E.; Tang, M.; Jensen, T.R.; Kjaer, K.; Bolbach, G.; Leiserowitz, L.; Weissbuch I.; Lahav, M. Science 2002, 295, 1266-1269.

[21] Nery, J.G.; Bolbach, G.; Weissbuch, I.; Lahav, M. Chem. Eur. J. 2005, 11, 3039-3048.

[22] Nery, J.G.; Eliash, R.; Bolbach, G.; Weissbuch, I.; Lahav, M. Chirality 2007, 19, 612-624.

[23] Rubinstein, I.; Eliash, R.; Bolbach, G.; Weissbuch, I.; Lahav, M. Angew. Chem. 2007, 119, 3784, Angew. Chem. Int. Ed. 2007, 46, 3710-3713.

[24] Rubinstein, I.; Clodic, G.; Bolbach, G.; Weissbuch, I.; Lahav, M. Chem. Eur. J. 2008, 14, 10999-11009.

[25] Illos, R.A.; Bisogno, F.R.; Clodic, G.; Bolbach, G.; Weissbuch, I.; Lahav, M. J. Am. Chem. Soc. 2008, $130,8651-8659$.

[26] Illos, R.A.; Clodic, G.; Bolbach, G.; Weissbuch, I.; Lahav, M. Orig. Life Evol. Biosph. 2010, 40, 51-63.

[27] Blanco, C.; Hochberg, D. Chem. Commun. 2012, 48, 3659-3661.

[28] Wattis, J.A.; Coveney, P.V. J. Phys. Chem. B 2007, 111, 9546-9562.

[29] Markvoort, A.J.; ten Eikelder, H.M.M.; Hilbers, P.A.J.; de Greef, T.F.A.; Meijer, E.W. Nat. Commun. 2011, 2:509-1/9, doi:10.1038/ncomms1517.

[30] Digital Library of Mathematical Functions. National Institute of Standards and Technology. http://dlmf.nist.gov/26 (accessed Feb 21, 2012).

[31] Rudin, W. Principles of Mathematical Analysis; McGraw-Hill: New York, 1976; Chap 10.

[32] Wagner, N.; Rubinov, B.; Ashkenasy, G. ChemPhysChem 2011, 12, 2771-2780.

[33] Brack, A. Chem. Biodiversity 2007, 4, 665-679.

[34] Maury, C.P.J. Orig. Life Evol. Biosph. 2009, 39, 141-150.

[35] Rubinov, B.; Wagner, N.; Rapaport, H.; Ashkenasy, G. Angew. Chem. Int. Ed. 2009, 48, 6683-6686.

[36] Williams, R.J.; Smith, A.M.; Collins, R.; Hodson, N.; Das, A.K.; Ulijn, R.V. Nat. Nanotecnol. 2009 , $4,19-24$. 
[37] Carnall, J.M.A.; Waudby, C.A.; Belenguer, A.M.; Stuart, M.C.A.; Peyralans, J.J.P.; Otto, S. Science 2010, 327, $1502-1506$.

[38] Joyce, G.F.; Visser, G.M.; van Boeckel, C.A.; van Boom, J.H.; Orgel, L.E.; van Westrenen, J. Nature 1984, 310, 602-604.

[39] Mills, W.H. J. Soc. Chem. Ind. 1932, 10, 750-759.

[40] Mislow, K. Collect. Czech. Chem. Commun. 2003, 68, 849-864.

[41] Green, M.M.; Garetz, B.A. Tetrahedron Letters 1984, 25, 2831-2834.

[42] Bolli, M.; Micura, R.; Eschenmoser, A. Chem. Biol. 1997, 4, 309-320.

[43] Siegel, J.S. Chirality, 1998, 10, 24-27. 